\title{
Nanoparticle-delivered TLR4 and RIG-I agonists enhance immune response to SARS-CoV-2 subunit vaccine
}

\author{
Alexandra Atalis*1, Mark C Keenum*1 ${ }^{\star 1}$ Bhawana Pandey ${ }^{1}$, Alexander Beach ${ }^{2}$, Pallab \\ Pradhan ${ }^{1,3,4}$, Casey Vantucci ${ }^{1}$, Ritika Jain ${ }^{1}$, Justin Hosten ${ }^{1}$, Clinton Smith ${ }^{1}$, Liana \\ Kramer $^{1}$, Angela Jimenez ${ }^{1}$, Miguel Armenta Ochoa ${ }^{1}$, David Frey ${ }^{1}$, Krishnendu Roy**1,3,4 \\ ${ }^{*}$ These authors contributed equally to this work \\ ${ }^{* *}$ Corresponding author email: krish.roy@gatech.edu \\ ${ }^{* *}$ Corresponding author mailing address: Krone Engineered Biosystems Building, 950 \\ Atlantic Drive NW, Atlanta, Georgia, 30332
}

1. Wallace H. Coulter Department of Biomedical Engineering Georgia Institute of Technology and Emory University Atlanta, Georgia, USA

2. School of Materials Science and Engineering Georgia Institute of Technology Atlanta, Georgia, USA

3. Marcus Center for Therapeutic Cell Characterization and Manufacturing Georgia Institute of Technology Atlanta, Georgia, USA

4. The Parker H. Petit Institute for Bioengineering and Biosciences Georgia Institute of Technology Atlanta, Georgia, USA

Keywords: COVID-19 protein subunit vaccine; SARS-CoV-2 spike protein; Combination adjuvant; Monophosphoryl lipid A; Intranasal versus intramuscular vaccination; Adaptive immune response 
bioRxiv preprint doi: https://doi.org/10.1101/2022.01.31.478507; this version posted February 2, 2022. The copyright holder for this preprint (which was not certified by peer review) is the author/funder. All rights reserved. No reuse allowed without permission.

\section{GRAPHICAL ABSTRACT}

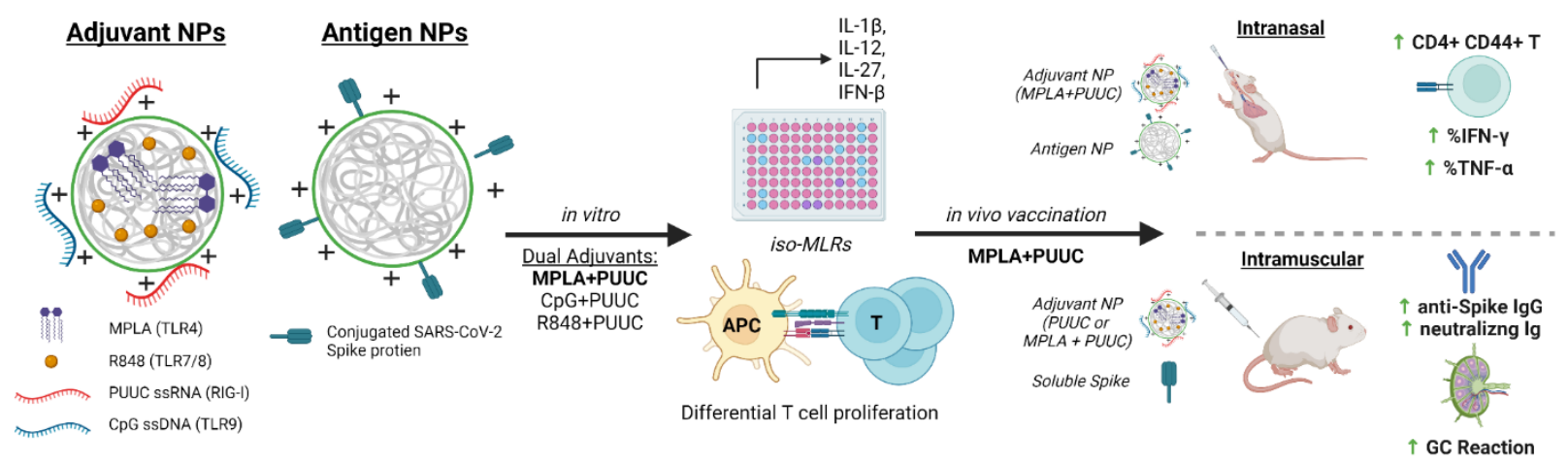




\section{ABSTRACT}

Despite recent success in vaccinating populations against SARS-CoV-2, concerns about immunity duration, continued efficacy against emerging variants, protection from infection and transmission, and worldwide vaccine availability, remain. Although mRNA, pDNA, and viral-vector based vaccines are being administered, no protein subunit-based SARSCoV-2 vaccine is approved. Molecular adjuvants targeting pathogen-recognition receptors (PRRs) on antigen-presenting cells (APCs) could improve and broaden the efficacy and durability of vaccine responses. Native SARS-CoV-2 infection stimulate various PRRs, including toll-like receptors (TLRs) and retinoic-acid-inducible gene I-like receptors (RIG-I). We hypothesized that targeting the same PRRs using adjuvants on nanoparticles along with a stabilized spike (S) protein antigen could provide broad and efficient immune responses. Formulations targeting TLR4 (MPLA), TLR7/8 (R848), TLR9 (CpG), and RIG-I (PUUC) delivered on degradable polymer-nanoparticles (NPs) were combined with the $\mathrm{S} 1$ subunit of $\mathrm{S}$ protein and assessed in vitro with isogeneic mixed lymphocyte reactions (iso-MLRs). For in vivo studies, the adjuvanted nanoparticles were combined with stabilized $S$ protein and assessed using intranasal and intramuscular prime-boost vaccination models in mice. Combination NP-adjuvants targeting both TLR and RIG-I (MPLA+PUUC, CpG+PUUC, or R848+PUUC) differentially increased proinflammatory cytokine secretion (IL-1 $\beta$, IL-12p70, IL-27, IFN- $\beta$ ) by APCs cultured in vitro, and induced differential $\mathrm{T}$ cell proliferation. When delivered intranasally, MPLA+PUUC NPs enhanced local CD4+CD44+ activated memory $T$ cell responses while MPLA NPs increased anti-S-protein-specific IgG and IgA in the lung. Following intramuscular delivery, PUUC-carrying NPs induced strong humoral immune responses, characterized by increases in anti-S-protein IgG and neutralizing antibody titers and germinal center B cell populations (GL7+ and BCL6+ B cells). MPLA+PUUC NPs further boosted $S$-protein-neutralizing antibody titers and $T$ follicular helper cell populations in draining lymph nodes. These results suggest that SARS-CoV-2-mimicking adjuvants and subunit vaccines could lead to robust and unique route-specific adaptive immune responses and may provide additional tools against the pandemic.

\section{INTRODUCTION}

The coronavirus disease 2019 (COVID-19) pandemic caused by severe acute respiratory syndrome coronavirus 2 (SARS-CoV-2) has killed more than 5 million people worldwide. ${ }^{1}$ The crisis has elicited a global scientific effort to develop vaccines and therapies for the disease at an unprecedented speed. Phase I trials started as early as March 2020 and as of November 2021, there at least 322 vaccines in various stages of development. ${ }^{2} \mathrm{~A}$ non-replicating viral vector vaccine from Janssen and two mRNA-based vaccines from Moderna and Pfizer-BioNTech - all against SARS-CoV-2 spike (S) protein antigen - have 
been authorized for emergency use in the United States. The Pfizer-BioNTech vaccine received full FDA approval for individuals 16 and older in August $2021 .{ }^{3}$ However, much remains to be determined regarding the longevity of immune responses and correlates of protection against emerging SARS-CoV-2 variant strains in various patient populations. Recently, booster shots, 2 months after initial dose of the Janssen vaccine and 6 months after initial dose of the mRNA vaccines, were approved due to waning immunity amongst vaccinated individuals. Much work remains to develop long term immunity and to understand how both local immunity in the lung and the associated systemic immunity can protect against emerging variants in a durable manner.

The incorporation of molecular adjuvants in vaccines, especially protein subunit vaccines (which make up $>35 \%$ of all vaccines in development), could be a potential strategy to induce more robust immune responses against SARS-CoV-2 through the targeting of receptors on antigen-presenting cells (APCs). ${ }^{2,4}$ Aluminum-containing adjuvants (e.g., alum) have been included in vaccines to enhance immunogenicity since the 1930s. ${ }^{5}$ Traditional adjuvants like alum have been developed and tested empirically, but in recent years vaccine design strategy has shifted to a more rational approach where each component elicits a defined immunological pathway to shift the immune response. Pathogen-associated molecular patterns (PAMPs), including lipids, carbohydrates, peptides, and nucleic acids commonly expressed by pathogens, are currently being investigated as adjuvants because they specifically bind to pattern-recognition receptors (PRRs) on APCs and induce maturation. ${ }^{4}$ Mature APCs, namely dendritic cells (DCs), initiate antigen-specific adaptive immune responses by activating naïve $T$ cells, which differentiate into effectors, such as cytotoxic T cells (CD8+) and T helper cells (CD4+). ${ }^{6}$ T helper cells (type 2, Th2) mediate the differentiation of B cells into antibody-producing plasma cells. ${ }^{7,8}$

Two classes of PRRs are toll-like receptors (TLRs) and retinoic-acid-inducible gene I (RIG-I)-like receptors. RIG-I receptors are in the cytosol and recognize short double stranded RNA, a replication intermediate for RNA viruses, that exhibit viral motifs such as an uncapped 5 ' diphosphate or triphosphate end. ${ }^{9}$ The poly-uridine core of Hepatitis $C$ virus (HCV), poly-U/UC (PUUC), activates RIG-I and triggers potent anti-HCV responses. ${ }^{10}$ The most studied TLR-ligand combination is TLR4 and the Gram-negative bacterial cell wall component lipopolysaccharide (LPS), which includes monophosphoryl lipid A (MPLA), a molecule used in FDA-approved adjuvant systems (AS01 and AS04, GlaxoSmithKline). ${ }^{11-13}$ Endosomal TLRs 7 and 8 recognize single-stranded RNA and can be activated by synthetic imidazoquinolines including resiquimod (R848). ${ }^{14}$ Endosomal TLR9 recognizes single-stranded DNA, and current understanding suggests that TLR9 recognizes unmethylated $\mathrm{CpG}$ motifs common to bacteria and viruses to discriminate from self-DNA. ${ }^{15}$ Most TLRs (TLR4, TLR7/8, TLR9) signal through the MyD88 pathway, which activates NFkB to induce production of proinflammatory cytokines (i.e., IL-1 $\beta$ ) and 
IRF7 to induce type I interferon secretion. ${ }^{16-18}$ When trafficked from the plasma membrane to endosomes, TLR4 can signal through the TRIF pathway and induce expression of IFN$\beta$ (Figure 1A). ${ }^{18,19}$

A single pathogen can express multiple PAMPs which concurrently stimulate multiple PRRs on immune cells. For instance, the live-attenuated yellow fever vaccine activates TLR2, TLR7, TLR8, and TLR9 on different DC subsets. Similarly, the live-attenuated Bacillus Calmette-Guérin (BCG) tuberculosis vaccine signals through TLR2, 4, and 9, among other PRRs. ${ }^{20}$ Positive-sense single-stranded RNA viruses like SARS-CoV-2 interact with TLR7 and TLR8, and produce dsRNA replication intermediates that are recognized by RIG-I and TLR3. ${ }^{21-24}$ TLR2 (dimerized with TLR1 or TLR6) and TLR4 have also exhibited potential to recognize viral proteins; in fact, SARS-CoV-2 S protein reportedly induces TLR4 signaling. ${ }^{25-27}$ Studies have shown that combinations of PAMPs result in synergistic, complementary, and antagonistic effects on innate and adaptive immunity. We have recently reported co-delivery of MPLA and CpG DNA on poly(lacticco-glycolic acid)-polyethylenimine (PLGA-PEI) particles induces prolonged IRF-5 phosphorylation, leading to synergistic increases in proinflammatory cytokine secretion in APCs. ${ }^{28}$ Further, we have demonstrated that intramuscular vaccination of R848, PUUC and $\mathrm{H} 1 \mathrm{~N} 1$ hemagglutinin on PLGA-PEI particles elevates CD8+ T cell populations in the lung and CD4+ antigen-specific T cell populations in the spleen in mice. ${ }^{29}$

To evaluate the effects of PRR-targeted adjuvants on SARS-CoV-2 protein subunit vaccination, we first formulated PLGA-PEI nanoparticles (NPs) with pairings of MPLA (TLR4), R848 (TLR7/8), or CpG DNA (TLR9) plus PUUC (RIG-I), and then combined the NPs with SARS-CoV-2 spike S1 subunit (containing the ACE-2 receptor binding domain) for in vitro studies or full stabilized $S$ protein for in vivo studies. The various formulations were tested in vitro using iso-mixed leukocyte reactions (iso-MLRs) and in vivo using intranasal and intramuscular prime-boost vaccination models. SARS-CoV-2 is an airborne virus that is mainly transmitted through contact with respiratory droplets from the nose and throat of an infected person ${ }^{30,31}$, resulting in severe lung infection and subsequent systemic infection (i.e., viremia) ${ }^{32}$. We explored both intranasal vaccination (I.N.) to induce local immune memory in the lung that could protect from SARS-CoV-2 infection and viremia as well as prevent transmission, and intramuscular (I.M.) vaccination to induce broad systemic immunity. We show that I.N. vaccination with MPLA+PUUC NP induced CD4+CD44+ activated memory $T$ cells in the lung while MPLA NPs produced anti-S protein IgG and IgA antibodies in the broncho-alveolar lavage (BAL) fluid. Interestingly, I.N. vaccination did not produce detectable antibodies in the serum. In contrast PUUC-carrying NPS, when delivered I.M. with various doses of the S protein, produced strong humoral responses, characterized by increased neutralizing antibody levels and germinal center (GC) B cells. Intramuscular vaccination of MPLA+PUUC NP generated similar responses to PUUC NP while further raising anti-spike protein 
neutralization titers and increasing $\mathrm{T}$ follicular helper cell responses in the draining lymph nodes (dLNs). Collectively, our data show route-specific polarization of local versus systemic immune responses against SARS-Cov2 using NP-adjuvanted protein subunit vaccines and suggest potential for inducing both lung-specific and systemic immunity by targeting I.N. vs I.M vaccination routes.

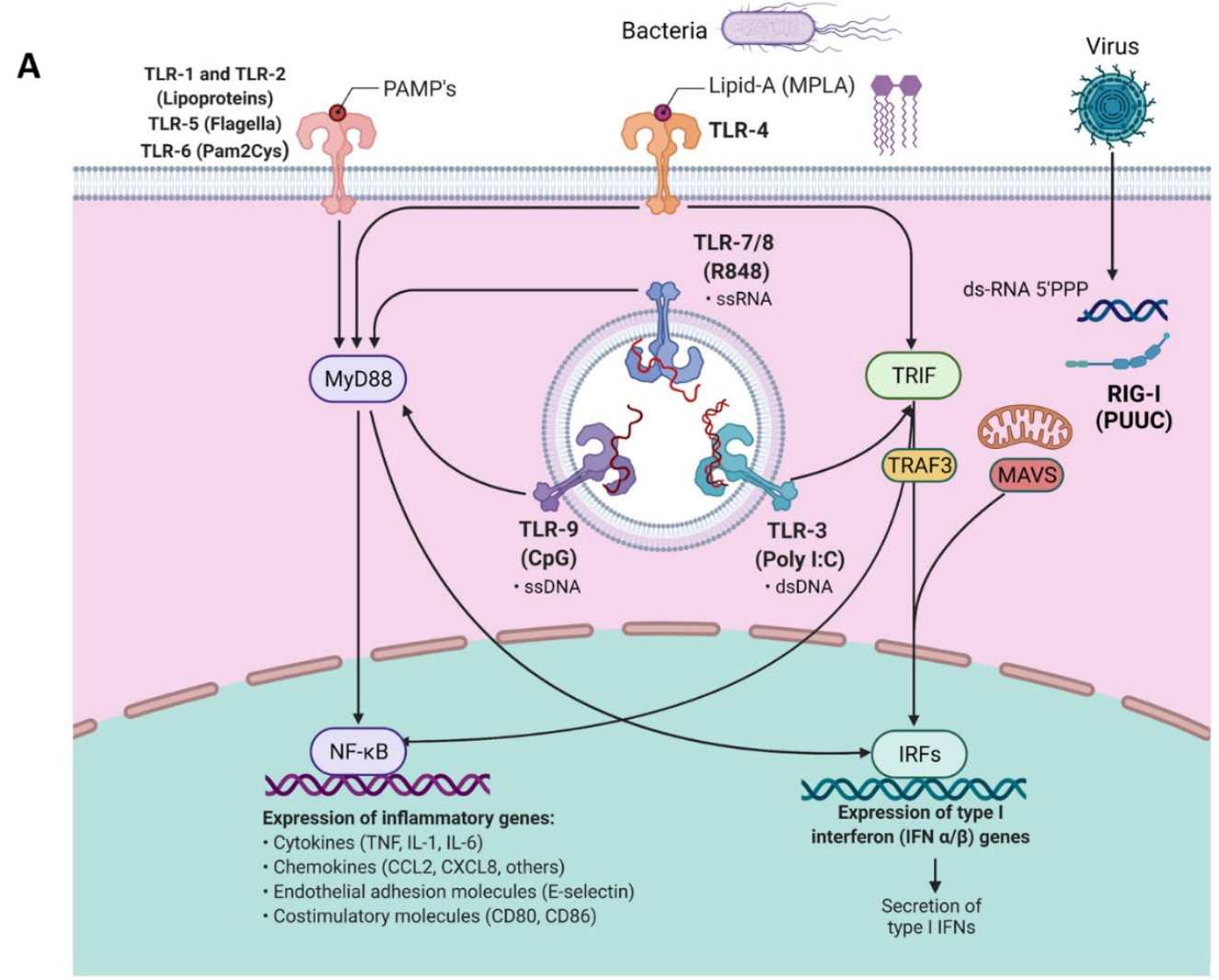

B

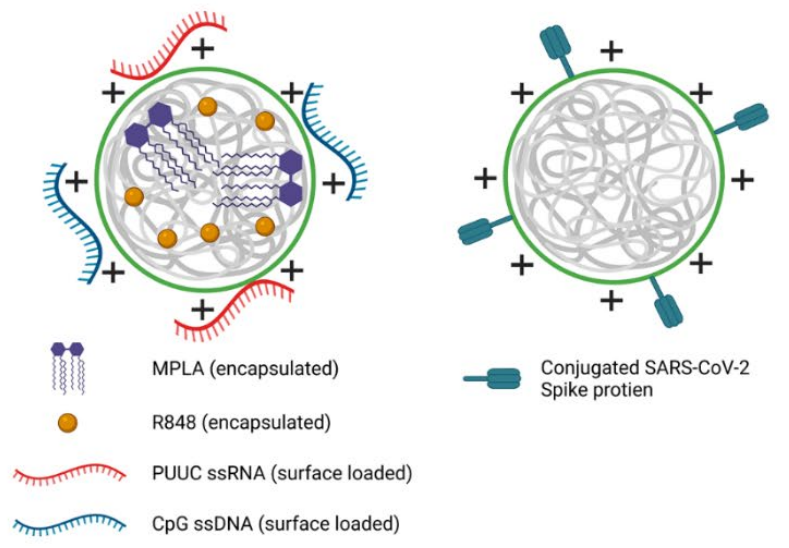

Figure 1: Multiple TLR and RLR intracellular signaling pathways intersect and can be activated by nanoparticles with encapsulated and surface-loaded pathogen-associated molecular patterns (PAMPs). A) Schematic of signaling downstream from TLR 1, TLRs 3-9, and 
RIG-I-like receptors indicating MyD88, TRIF, TRAF3, and MAVS as important intermediates leading to nuclear translocation of NF-kB or IRFs to synthesize proinflammatory genes. B) Depiction of poly(lactic-co-glycolic acid)-polyethyleneimine nanoparticles (PLGA-PEI NPs) with encapsulated hydrophobic molecules (R848, MPLA) and/or surface loaded nucleic acids (CpG, PUUC) for adjuvant delivery or surface-loaded SARS-CoV-2 spike protein for antigen delivery.

\section{MATERIALS AND METHODS}

\section{Synthesis of PUUC (RIG-I Agonist)}

The RIG-I agonist poly-U/UC (PUUC) is based on a Hepatitis C Virus (HCV) RNA sequence. ${ }^{10}$ PUUC RNA was transcribed from custom DNA templates (Integrated DNA Technologies, Custom PAGE-purified Ultramer oligos) with the MEGAshortscript T7 Transcription Kit (Invitrogen, Cat\# AM1354) as previously described. ${ }^{19}$ The templates were: 5'-TAA TAC GAC TCA CTA TAG GCC ATC CTG TTT TTT TCC CTT TTT TTT TTT CTT TTT TTT TTT TTT TTT TTT TTT TTT TTT TTT TTC TCC TTT TTT TTT CCT CTT TTT TTC CTT TTC TTT CCT TT-3' (forward) and 5'-AAA GGA AAG AAA AGG AAA AAA AGA GGA AAA AAA AAG GAG AAA AAA AAA AAA AAA AAA AAA AAA AAA AAA AAA AGA AAA AAA AAA AGG GAA AAA AAC AGG ATG GCC TAT AGT GAG TCG TAT TA$3^{\prime}$ (reverse). PUUC was purified by chilled ethanol precipitation followed by centrifugation and resuspension in nuclease free water (Boston BioProducts, Cat\# R-100DR) at 0.5 to $1.0 \mathrm{mg} / \mathrm{mL}$, and yields were quantified the Nucleic Acid Quantification workflow on a Synergy HT plate reader (BioTek) with Gen5 software. Synthesized PUUC was stored at $-80^{\circ} \mathrm{C}$ until use.

\section{PLGA-PEI Nanoparticle Synthesis and Adjuvant Loading}

PLGA nanoparticles were synthesized via a double-emulsion and solvent evaporation method as previously reported. ${ }^{28,29,33}$ PLGA (50:50, MW: 7,000-17,000, Resomer RG $502 \mathrm{H}$, Sigma-Aldrich, Cat\# 719897) was dissolved in dichloromethane (DCM, SigmaAldrich, Cat\# 270997) in 1:20 w/v ratio. Endotoxin-free water was added in a 1:4 v/v ratio to the mixture to form the first water-oil emulsion. For particles with encapsulated adjuvant, either R848 (4.80-6.67 $\mu \mathrm{g} / \mathrm{mg}$ PLGA, STEMCELL Technologies, Cat\# 73784) or MPLA PHAD® $(1 \mu \mathrm{g} / \mathrm{mg}$ PLGA, Avanti Polar Lipids, Cat\# 699800P) was dissolved the DCM added to the first water-oil emulsion. The first emulsion was sonicated for $2 \mathrm{~min}$ at $65 \%$ power at RT, and then added to 5\% PVA (MW: 31,000-50,000) in water at a 5:16 $\mathrm{v} / \mathrm{v}$ ratio to form a second water-oil-water emulsion. The second emulsion was sonicated at $65 \%$ power for $5 \mathrm{~min}$ at RT. DCM was evaporated by stirring the second emulsion at $\mathrm{RT}$ for $3 \mathrm{~h}$. Large PLGA aggregates were removed by centrifugation at $2000 \times \mathrm{g}$ for 10 min. The supernatant was then ultracentrifuged at $80,000 \times \mathrm{g}$ for $20 \mathrm{~min}$ to pellet the PLGA NPs. Nanoparticles were washed with DI water via ultracentrifugation, resuspended in DI water and lyophilized for $48 \mathrm{~h}$. Branched polyethyleneimine (bPEl, 
Polysciences, Cat\# 06090) was coated onto the PLGA nanoparticles by EDC (Thermo Scientific, Cat\# 22980) and sulfo-NHS (Thermo Scientific, Cat\# PG82071) chemistry to produce cationic PLGA-PEI nanoparticles. PLGA NPs were resuspended in $0.1 \mathrm{M}$ aqueous MES (Sigma-Aldrich, Cat\# M5287) and 40 molar excess of EDC and 25 molar excess of sulfo-NHS were added to the suspension. After end-to-end rotation for $2 \mathrm{~h}$, a $1: 2 \mathrm{v} / \mathrm{v}$ bPEI solution in 0.2 MES was added to the particle suspension. After stirring for 2 $\mathrm{h}$, particles were ultracentrifuged at $80,000 \times \mathrm{g}$ twice in $1 \mathrm{M} \mathrm{NaCl}$ and once in $\mathrm{DI}$ water. PLGA-PEI NPs were resuspended in DI water and lyophilized for $48 \mathrm{~h}$. For particles electrostatically loaded with nucleic acid adjuvants, either Class B CpG ODN 1826 (Invitrogen, Cat\# tIrl-1826) or PUUC was incubated with particles in $10 \mathrm{mM}$ sodium phosphate buffer (made with nuclease free water) under end-to-end rotation for $24 \mathrm{~h}$.

Nanoparticle size and surface zeta potential were measured with a Zetasizer Nano ZS (Malvern). R848 loading was determined by dissolving particles in sterile-filtered DMSO (Tocris, Cat\# 3176) followed by absorbance readings against a standard curve at 324 $\mathrm{nm}$. MPLA loading estimation via GC-MS, LC-MS, and surrogate fluorometry has been previously described. ${ }^{28}$ PUUC and $\mathrm{CpG}$ loading was quantified by supernatant measurement of unbound RNA or DNA, respectively, with the Nucleic Acid Quantification workflow on a Synergy HT plate reader (BioTek) with Gen5 software.

\section{Spike-Conjugated Nanoparticle Synthesis}

To conjugate SARS-CoV-2 spike to PLGA-PEI NPs, free amines of particles were converted to thiols via Traut's reagent. Initially, PLGA-PEI NPs were dispersed in $100 \mathrm{mM}$ phosphate buffer with EDTA $(2 \mathrm{mM})(\mathrm{pH}=8.0)$ and mixed with excess amount of 2iminothiolane (Traut's reagent, Sigma-Aldrich, Cat\# 16256). After continuous rotation overnight at RT, thiolated PLGA-PEI (PLGA-PEI-SH) NPs were purified by centrifugation, washing and lyophilization overnight. Thiol groups on particles were estimated by Ellman's reagent ( $G$ Biosciences, Cat\# BC87) using cysteine (Sigma-Aldrich, Cat\# 168149 ) as the standard, yielding a $60-70 \%$ thiolation efficiency. Until spike loading, PLGA-PEI-SH NPs were stored at $-20^{\circ} \mathrm{C}$. Prior to in vivo experiments, a stock solution of NHS-PEG-SPDP (bifunctional crosslinker, Sigma-Aldrich Cat\# 803499) was prepared in anhydrous DMSO (10 mg/mL, Sigma-Aldrich, Cat\# 276855). Further, excess NHS-PEGSPDP $(200 \mu \mathrm{g})$ crosslinker was added to well-dispersed PLGA-PEI-SH NPs solution in PBS (5 mg/mL, pH = 7.6) with stabilized SARS-CoV-2 spike glycoprotein $(10 \mu \mathrm{g} / \mathrm{mg} \mathrm{NP}$, with Avi Tag, BEI Resources, Cat\# NR-53524). Following rotation for $6 \mathrm{~h}$ at RT, NPs were centrifuged at 21,000 x $g$ for $10 \mathrm{~min}$, and supernatant was saved (first wash). NPs were washed with nuclease free water, centrifuged, and supernatant was also saved (second wash). Supernatant washes were filtered through a 100,000 MWCO Ultra-4 Amicon centrifugal filter (Millipore Sigma, Cat\# UFC810096) to retain unconjugated spike glycoprotein and separate remaining NHS-PEG-SPDP crosslinker. Unconjugated spike protein was measured via micro-BCA (Boster Bio, Cat\# AR1110). 


\section{Bone Marrow Derived Dendritic Cell (BMDC) Culture}

GM-CSF-derived BMDCs were generated similarly to other reports. ${ }^{34}$ Female Balb/cJ (510 weeks old, Jackson Labs) were euthanized with $\mathrm{CO}_{2}$. PBS was flushed through tibiae and femurs to isolate bone marrow, which was strained through a $40 \mu \mathrm{m}$ strainer. Bone marrow cells were centrifuged, treated in RBC lysis buffer (Thermo Fisher, Cat\# 00-433357 ), and were seeded in 100-mm round tissue-culture treated polystyrene dishes at 1 million cells/mL in complete RPMI medium (with L-glutamine, Gibco, Cat\# 11875119) with $10 \%$ characterized fetal bovine serum (GE Healthcare, Cat\# SH30071.03), 1\% penicillinstreptomycin (Corning, Cat\# 30-002-CI), 1xß-mercaptoethanol (Thermo Fisher, Cat\# 21985023), $1 \mathrm{mM}$ sodium pyruvate (Thermo Fisher, Cat\# 11360070), and $20 \mathrm{ng} / \mathrm{mL}$ murine GM-CSF (Peprotech, Cat\# 315-03). Media was replenished on days 2, 4, and 6 of culture, and experiments with GM-CSF BMDCs were conducted on day 7 of culture. For FLT3L BMDCs, RBC-lysed murine bone marrow was seeded at 2 million cells/mL in complete RPMI media with $200 \mathrm{ng} / \mathrm{mL}$ human FLT3 ligand (PeproTech, Cat\# 300-19) in a 6-well plate (10 million cells/well). Experiments with FLT3L BMDCs were conducted on day 9 of culture.

\section{Characterization of APC Subsets in BMDC Culture}

Both FLT3L and GM-CSF BMDCs were stained with 1:1000 Zombie Green Fixable Viability Kit (BioLegend, Cat\# 423111) and were blocked with anti-mouse CD16/32 (BioLegend, Cat\# 101320) and True-Stain Monocyte Blocker (BioLegend, Cat\# 426102). After blocking, cells were stained with anti-mouse CD11b (BUV395, BD, Cat\# 563553), CD11c (Brilliant Violet 421, Biolegend, Cat\# 117343), B220 (APC, Biolegend, Cat\# 103212), Ly-6C (Brilliant Violet 711, Biolegend, Cat\# 128037), Ly-6G (Brilliant Violet 785, Biolegend, Cat\# 127645), CD64 (PE, Biolegend, Cat\# 139304), F4/80 (PE/Cy5, Biolegend, Cat\# 123112), and MHC-II (APC/Cy7, Biolegend, Cat\# 107628). Cells were fixed with BD Cytofix (Cat\# 554655) and analyzed with a BD LSRFortessa flow cytometer. FlowJo Software (BD) was used to generate t-distributed stochastic neighbor embedding (t-SNE) plots.

\section{In Vitro Activation of BMDCs and Iso-Mixed Lymphocyte Reaction (Iso-MLR)}

Adjuvanted PLGA-PEI NPs and the S1 subunit of the SARS-CoV-2 spike protein (Novus Biologicals, Cat\# NBP2-90985, 100 ng protein/500,000 cells/mL RPMI media) were added to GM-CSF or FLT3L-cultured BMDCs in a U-bottom 96 well plate. Each well contained 100,000 BMDCs in 200 mL media. Doses of adjuvants are outlined in Table 1. The concentration of particles was adjusted based on the adjuvant dose and the particleonly ("blank") control was matched to the highest PLGA-PEI NP concentration to ensure NPs were not activating BMDCs. The cells were incubated with adjuvant-NPs for 24 hours before addition of $T$ cells. To isolate $T$ cells, spleens from female Balb/cJ mice were dissociated in $2 \mathrm{mg} / \mathrm{mL}$ Collagenase D (Sigma-Aldrich, Cat\# 11088882001) in Opti-MEM 
media (Gibco, Cat\# 11058021) and filtered through a $40 \mu \mathrm{m}$ cell strainer. T cells were magnetically separated from other splenocytes using the Mouse Pan-T Cell Isolation Kit II (Miltenyi Biotec, Cat\# 130-095-130), labeled with CellTrace ${ }^{\mathrm{TM}}$ CFSE (Thermo Fisher, Cat\# C34554), and resuspended in complete RPMI medium at 1 million cells/mL. BMDCPLGA NP U-bottom plates were centrifuged at $500 \times \mathrm{g}$ for 5 minutes and supernatants were collected and frozen at $-20^{\circ} \mathrm{C}$. $200,000 \mathrm{~T}$ cells were added to each well containing BMDCs. After 72 hours, $T$ cells were stained for flow cytometric analysis with the following: Zombie UV (Biolegend, Cat\# 423107) to exclude dead cells, and stained with anti-mouse CD3 (Brilliant Violet 786, Biolegend), anti-mouse CD4 (APC, Biolegend) and anti-mouse CD8a (PE, Biolegend) to identify CD4+ and CD8+ T cells. A well containing only CSFE-stained T cells was included as a non-proliferative control. Percentages of proliferating $T$ cells were calculated by gating on the reduced FITC signal (Figure S1). DuoSet ELISA kits (R\&D Systems) were used to quantify IFN- $\beta$ and IFN- $\lambda 3$ from BMDC supernatants. IL-12p70, IL-1 $\beta$, and IL-27 were quantified using Luminex $®$ assays (R\&D systems). Supernatants were diluted 4-fold for all cytokines except for IL-1 $\beta$, in which case they were diluted 8-fold. Supernatants were analyzed in triplicate for each experimental condition.

\begin{tabular}{|c|c|c|c|}
\hline $\begin{array}{l}\text { Particle } \\
\text { Adjuvants }\end{array}$ & $\begin{array}{l}\text { Adjuvant } \\
\text { combinations }\end{array}$ & $\begin{array}{c}\text { Loading level } \\
(\mu \mathrm{g} / \mathrm{mg} \text { PLGA-PEI NP) }\end{array}$ & $\begin{array}{l}\text { Adjvuant Dose for BMDCs } \\
\text { (ng/500,000 cells } / \mathrm{mL})\end{array}$ \\
\hline PLGA-CpG & CpG & 60 & 500 \\
\hline PLGA-MPLA & MPLA & 6 & 100 \\
\hline PLGA-PUUC & PUUC & 6 & 100 \\
\hline PLGA-R848 & $\mathrm{R} 848$ & 4.8 & 100 \\
\hline \multirow{2}{*}{$\begin{array}{c}\text { PLGA- } \\
\text { CpG+PUUC }\end{array}$} & CpG & 30 & 500 \\
\hline & PUUC & 6 & 100 \\
\hline \multirow{2}{*}{$\begin{array}{c}\text { PLGA- } \\
\text { MPLA+CpG }\end{array}$} & MPLA & 6 & 50 \\
\hline & CpG & 60 & 500 \\
\hline \multirow{2}{*}{$\begin{array}{c}\text { PLGA- } \\
\text { MPLA+PUUC }\end{array}$} & MPLA & 6 & 100 \\
\hline & PUUC & 6 & 100 \\
\hline PLGA- & R848 & 4.8 & 100 \\
\hline R848+PUUC & PUUC & 5 & 100 \\
\hline
\end{tabular}

Table 1. Single and combination adjuvant loading on PLGA NP and doses for GM-CSF and FLT3L BMDCs in iso-MLR assays.

\section{In Vivo Intranasal Vaccination}

For this experiment, there were six mice per treatment and control group for a total of 78 mice in thirteen groups: (A) Seven vaccine groups included $1 \mu \mathrm{g}$ of unformulated SARSCoV-2 spike protein (R\&D Systems, Cat\# 10549-CV-100) delivered with adjuvanted NPs: MPLA $(24 \mu \mathrm{g})$, CpG (20 $\mu \mathrm{g})$, PUUC (17 $\mu \mathrm{g})$, CpG-PUUC (20 $\mu \mathrm{g}, 17 \mu \mathrm{g})$, MPLA-PUUC 
$(20 \mu \mathrm{g}, 17 \mu \mathrm{g}), \mathrm{R} 848-P U U C(20 \mu \mathrm{g}, 20 \mu \mathrm{g})$, and MPLA-CpG $(24 \mu \mathrm{g}, 20 \mu \mathrm{g})$. (B) Two vaccine groups included $1 \mu \mathrm{g}$ of PLGA NP-conjugated SARS-CoV-2 spike protein (methods described above) delivered with adjuvanted NPs: PUUC (17 $\mu \mathrm{g})$ and MPLAPUUC (20 $\mu \mathrm{g}, 17 \mu \mathrm{g})$. (C) There were three controls included: saline, unformulated SARSCoV-2 spike only $(1 \mu \mathrm{g})$ and PLGA NP-conjugated SARS-CoV-2 spike only $(1 \mu \mathrm{g})$. (D) One vaccine group included $2 \mu \mathrm{g}$ of unformulated stabilized SARS-CoV-2 spike protein delivered with MPLA-PUUC PLPs $(20 \mu \mathrm{g}, 17 \mu \mathrm{g}$ ). On days 0 (prime) and 28 (boost), adjuvant-loaded NPs and either unformulated spike protein or spike-conjugated NPs were resuspended in normal saline and administered to 9-10-week-old female BALB/c mice dropwise in the bilateral nares (4 mg NPs and $1 \mu \mathrm{g}$ antigen in $60 \mu \mathrm{L}$ saline per mouse). Half of each experimental group (3 mice) was euthanized at day 27, and blood and bronchoalveolar lavage (BAL) fluid was collected. The remaining 3 mice in each group were euthanized at day 35, and blood, BAL fluid, and lungs were collected. All blood samples were clotted for 30-60 min at RT in serum separator tubes (BD, Cat\# 365967), and sera were isolated by centrifugation at $4,000 \times \mathrm{g}$ for $15 \mathrm{~min}$ at $4{ }^{\circ} \mathrm{C}$. Sera were heat inactivated at $56{ }^{\circ} \mathrm{C}$ for $30 \mathrm{~min}$ in a water bath to inhibit complement binding and then stored at $-80^{\circ} \mathrm{C}$ until use. BAL fluid samples were centrifuged to remove cells and stored at $-80^{\circ} \mathrm{C}$.

\section{Ex Vivo Lung Cell Restimulation}

Harvested lungs from intranasally vaccinated mice were processed into single cell suspensions with a gentleMACS ${ }^{\mathrm{TM}}$ Octo Dissociator and Lung Dissociation Kit (Miltenyi Biotec) according to manufacturer's instructions including RBC lysis. Cells were centrifuged and resuspended at 10 million cells $/ \mathrm{mL}$ in RPMI media with $10 \%$ FBS, $1 \%$ penicillin-streptomycin, $1 \mathrm{mM}$ sodium pyruvate, and $1 \mathrm{x} \beta$-mercaptoethanol. Cells were seeded at 2.4 million cells per well in a treated 96-well plate and left to culture overnight. Lung cells were centrifuged and resuspended with fresh media with $20 \mu \mathrm{L} / \mathrm{mL}$ of PeptTivator ${ }^{8}$ SARS-CoV-2 Prot_S (Miltenyi Biotec) and $5 \mu \mathrm{g} / \mathrm{mL}$ Brefeldin A (BioLegend). After $6 \mathrm{~h}$, cells were stained for 30 min at RT with Zombie Green ${ }^{\mathrm{TM}}$ Fixable Viability Kit (BioLegend) and were blocked with anti-mouse CD16/32 (BioLegend) and True-Stain Monocyte Blocker ${ }^{\mathrm{TM}}$ (BioLegend). Following blocking, cell surfaces were stained for $30 \mathrm{~min}$ at $4^{\circ} \mathrm{C}$ with anti-mouse CD45 (BD, BUV395), CD44 (Biolegend, BV711), CD69 (Biolegend, BV785), CD103 (Biolegend, PE-Dazzle 594), CD8a (Biolegend, PE/Cy5), and CD4 (Biolegend APC). Surface-stained cells were fixed and permeabilized for 30 min at $4^{\circ} \mathrm{C}$ with the Foxp3/Transcription Factor Staining Buffer Set (eBioscience). Then cells were stained with anti-mouse IFN-y (PE), Granzyme B (Pacific Blue), and TNF- $\alpha$ (PE/Cy7). Staining was measured with a BD LSRFortessa ${ }^{\text {TM }}$ flow cytometer. 
In Vivo Intramuscular Vaccination and Analysis of Popliteal Lymph Nodes

NPs were loaded with MPLA (6 $\mu \mathrm{g} / \mathrm{mg} \mathrm{NP}$ ), PUUC (5 $\mu \mathrm{g} / \mathrm{mg}$ NP), or MPLA+PUUC. Female BALB/c mice were intramuscularly injected with adjuvant-loaded NPs (4 $\mathrm{mg} / \mathrm{mouse}$ ) and variable doses of stabilized SARS-CoV-2 spike glycoprotein (BEI Resources, Cat\# NR-52397) into the left and right anterior tibialis anterior muscles (50 $\mu \mathrm{L}$ NPs in saline per injection) on day 0 (prime) and day 28 (boost). On day 36, mice were euthanized, and blood, bilateral popliteal lymph nodes, and spleens were harvested. Blood was processed as described above to isolate sera. Lymph nodes from each leg per mouse were combined and passed through a $40 \mu \mathrm{m}$ cell strainer to generate single cell suspensions which were centrifuged and washed with PBS. Lymph node cells were stained with Zombie Green ${ }^{\mathrm{TM}}$ (Biolegend) and blocked with anti-mouse CD16/32 as above. After blocking, cell surfaces were stained for $30 \mathrm{~min}$ at $4^{\circ} \mathrm{C}$ with anti-mouse B220 (Biolegend, APC), GL7 (Biolegend, PE/Cy7), and CXCR5 (Biolegend, BV421). Surfacestained lymph node cells were then fixed, permeabilized and intracellularly stained with anti-mouse BCL6 (Biolegend, PE) in permeabilization buffer. Lymph node cells were fixed and analyzed with a BD FACSymphony ${ }^{\mathrm{TM}}$ A5 Cell Analyzer. The same experiment was repeated using spike-conjugated nanoparticles instead of unformulated spike protein with $1000 \mathrm{ng}$ spike protein/mouse delivered on days 0 and 28 for prime-boost regimen.

\section{Ex Vivo Splenocyte Restimulation}

Harvested spleens from intramuscularly vaccinated mice were processed into single cell suspensions with a gentleMACS ${ }^{\mathrm{TM}}$ Octo Dissociator and Spleen Dissociation Kit (Miltenyi Biotec) with slight modifications to the manufacturer's protocol. Specifically, to protect splenocyte viability, we ensured that tissue processing began within approximately 30 min of harvesting. As soon as samples were counted, samples were immediately centrifuged and resuspended at 10 million cells $/ \mathrm{mL}$ in BMDC media. Cells were seeded at 2 million cells per well in a treated 96 -well plate and left to culture overnight at $37^{\circ} \mathrm{C}$ in $5 \% \mathrm{CO}_{2}$. Cells were centrifuged and resuspended with fresh media with $40 \mu \mathrm{L} / \mathrm{mL}$ of PeptTivator® SARS-CoV-2 Prot_S (Miltenyi Biotec, Cat\# 130-126-700), $1 \mu \mathrm{L} / \mathrm{mL}$ monensin (BioLegend), and $5 \mu \mathrm{g} / \mathrm{mL}$ Brefeldin A (BioLegend). After $6 \mathrm{~h}$ of incubation, splenocytes were stained for 30 min at RT with Zombie Green ${ }^{\mathrm{TM}}$ Fixable Viability Kit (BioLegend) and were blocked with anti-mouse CD16/32 (BioLegend). Following blocking, cell surfaces were stained for $30 \mathrm{~min}$ at $4^{\circ} \mathrm{C}$ with anti-mouse CD8a (Biolegend, PE/Cy5), CD4 (Biolegend, APC), CD3 (BD, BUV395), CD44 (Biolegend, BV711). Surface-stained cells were fixed and permeabilized for $30 \mathrm{~min}$ at $4^{\circ} \mathrm{C}$ with the Foxp3/Transcription Factor Staining Buffer Set (eBioscience). Then cells were stained with anti-mouse IFN-y (Biolegend, PE), Granzyme B (Biolegend, BV421), IL-4 (Biolegend, $\mathrm{PE} /$ Dazzle 594) and TNF- $\alpha$ (Biolegend, PE/Cy7). Staining was measured with a BD FACSymphony ${ }^{\text {TM }}$ A5 Cell Analyzer. 


\section{ELISA Assay for Quantifying Anti-Spike Antibody Responses}

SARS-CoV-2 stabilized spike (BEI Resources, Cat\# NR-52397) was diluted $1 \mu \mathrm{g} / \mathrm{mL}$ in $0.05 \mathrm{M}$ carbonate-bicarbonate buffer ( $\mathrm{pH}$ 9.6). Diluted spike was adsorbed onto Nunc ${ }^{\mathrm{TM}}$ MaxiSorp $^{\mathrm{TM}}$ ELISA Plates by incubating $100 \mathrm{ng} /$ well overnight at $4^{\circ} \mathrm{C}$. Antigen-coated plates were washed three times with wash PBST (0.01 M PBS + 0.05\% Tween-20), and plates were blocked for $6-8 \mathrm{~h}$ at $4^{\circ} \mathrm{C}$ with PBSTBA (PBST $+1 \% \mathrm{BSA}+0.02 \% \mathrm{NaN}_{3}$ ). Blocked plates were incubated overnight at $4^{\circ} \mathrm{C}$ with $10^{2}-$ to $10^{6}$-fold diluted BAL fluid or sera from intranasally or intramuscularly vaccinated mice, respectively. Plates were washed three times with PBST. A secondary biotinylated anti-mouse $\lg A$, total $\lg \mathrm{G}, \lg \mathrm{G} 1$, or IgG2a antibody (SouthernBiotech) was diluted 5,000-fold in 5-fold diluted PBSTBA and was added to plates for $2 \mathrm{~h}$ at RT. Plates were similarly washed with PBST. 5,000-fold diluted streptavidin-conjugated horseradish peroxidase (strep-HRP, ThermoFisher) was incubated for $2 \mathrm{~h}$ at RT. Plates were washed six times. Ultra TMB-ELISA Substrate Solution (ThermoFisher) was incubated for 15 to $25 \mathrm{~min}$ for color to develop on the plate. Lastly, $2 \mathrm{~N}$ sulfuric acid was added to each well, and absorbance was measured at 450 and $630 \mathrm{~nm}$ on a Synergy HT plate reader (BioTek) with Gen5 software.

\section{Modified ELISA Assay to Measure Anti-Spike Neutralizing Antibodies}

Neutralizing antibodies were quantified like the ELISA method described above in a 384well UltraCruz ${ }^{\circledR}$ ELISA high-binding plate (Santa Cruz Biotechnology). Diluted spike in $0.05 \mathrm{M}$ carbonate-bicarbonate buffer (1 $\mathrm{gg}$ spike $/ \mathrm{mL}, \mathrm{pH} 9.6)$ was incubated in wells of a 384-well plate (50 ng/well) overnight at $4^{\circ} \mathrm{C}$. Plates were washed three times with PBST, and plates were blocked in PBSTBA for $6-8 \mathrm{~h}$ at $4^{\circ} \mathrm{C}$. Blocked plates were incubated overnight at $4^{\circ} \mathrm{C}$ with $10 \mathrm{e} 2$ - to $10 \mathrm{e} 6$-fold diluted sera from intramuscularly vaccinated mice. Plates were similarly washed. In lieu of a secondary antibody, plates were incubated for $2 \mathrm{~h}$ at RT with $500 \mathrm{ng} / \mathrm{mL}$ ( $25 \mathrm{ng} /$ well) recombinant biotinylated human ACE2 (R\&D Systems, Cat\# BT933-020) diluted in PBSTBA. Plates were washed with PBST, and 5,000-fold diluted strep-HRP (ThermoFisher) was incubated for $2 \mathrm{~h}$ at RT. Plates were washed again six times, and incubated with Ultra TMB-ELISA Substrate Solution (ThermoFisher) for $20 \mathrm{~min}$. The reaction was stopped with $2 \mathrm{~N}$ sulfuric acid, and absorbance was measured at 450 and $630 \mathrm{~nm}$ on a Synergy HT plate reader (BioTek) with Gen5 software. Absorbances were normalized by row to correct for plate-based effects.

\section{Statistical Analysis}

All flow cytometry FCS files were analyzed with FlowJo (v10, BD). Statistical analyses were performed with GraphPad Prism 9. Normality was assessed with the KolmogorovSmirnov test with Dallal-Wilkinson-Lilliefors $P$ value. For more than two comparisons, statistical differences between normally distributed datasets were determined with a one- 
way ANOVA with Tukey's post-hoc test for multiple comparisons. Similarly, nonparametric datasets were evaluated with the Kruskal-Wallis test and Dunn's post-hoc test. For antibody quantification with ELISA, area under the curve (AUC) across fold dilutions was computed with the AUC function on GraphPad as previously reported. ${ }^{35}$

\section{RESULTS}

TLR- and RIG-I-targeted combination adjuvants differentially induce GM-CSF BMDC proinflammatory cytokine secretion and FLT3L BMDC activation of T cells when delivered with SARS-CoV-2 S1 subunit protein

Hydrophobic R848 or MPLA were encapsulated into PLGA NP using a w/o/w emulsionsolvent evaporation method previously published. Blank particles without adjuvants were also prepared as a negative control. The average diameter of PLGA NPs prior to PEI modification was approximately $250 \mathrm{~nm} .^{28,29,33}$ To electrostatically load CpG DNA and PUUC RNA, PLGA NPs were modified with surface bPEI to produce cationic particles with an approximate zeta potential of $30 \mathrm{mV} .^{28,29,33}$ To assess if adjuvants are more immunostimulatory when paired with another or delivered alone, combination adjuvants MPLA+PUUC, CpG+PUUC, and R848+PUUC on NPs as well as single adjuvant and unformulated (Blank) control NPs were mixed with SARS-CoV-2 S1 subunit in media and incubated (adjuvant and S1 doses in Experimental Methods section) with murine BMDCs generated using either GM-CSF or FLT3L cytokines in a 96 well plate. After 24 hours, supernatants were collected to quantify BMDC proinflammatory cytokine secretion, and then $T$ cells were added for another 72 hours with activated BMDCs before quantifying $T$ cell proliferation using CellTrace CFSE.

Flow cytometry and tSNE analysis revealed Day 9 FLT3L-derived BMDC cultures were composed of conventional dendritic cells (cDC; CD11 $\mathrm{c}^{+} \mathrm{MHCl}{ }^{\mathrm{hi}} \mathrm{CD} 64^{\mathrm{lo} /}-\mathrm{F} 4 / 80^{\mathrm{lo} /}-$ ) and plasmacytoid dendritic cells (pDC; B220+Ly6C+CD11 ${ }^{+} \mathrm{MHCll}^{\mathrm{lo}} \mathrm{Ly}_{6 \mathrm{G}}^{\mathrm{lo}}$ ), whereas Day 7 GM-CSF-derived BMDCs were primarily monocytes (Ly6ChiCD11c-) and monocytederived DCs/macrophages (MoDC; $\mathrm{CD} 64^{+} \mathrm{F} 4 / 80^{+}$). There is some macrophage survival in FLT3L culture, and neutrophil (Ly6G ${ }^{\text {hi }} \mathrm{Ly}_{6 C^{+}}$) survival in GM-CSF culture (Figure 2A). MPLA+PUUC NPs statistically increased IL-1 $\beta$ and IL-27 secretion, R848+PUUC NPs upregulated IL-12p70 and IL-27, and CpG+PUUC NPs increased production of IFN- $\beta$ in GM-CSF BMDC culture compared to single adjuvant NP, blank NP, and antigen-only controls (Figure 2B-F). In FLT3L BMDC culture, however, CpG NPs increased and $\mathrm{CpG}+\mathrm{PUUC}$ NPs antagonistically decreased IFN- $\lambda 3$ secretion (Figure 2G).

Next, we evaluated the ability of combination adjuvant-activated BMDCs to stimulate murine $\mathrm{T}$ cell proliferation in iso-mixed lymphocyte (iso-MLR) reactions. After $72 \mathrm{~h}$ there were no significant changes in CD4 T cell proliferation with MPLA- or PUUC-stimulated 
GM-CSF BMDCs while CpG, R848, MPLA-CpG, CpG+PUUC, MPLA+PUUC and R848+PUUC-stimulated GM-CSF BMDCs induced two-fold decreases in proliferation compared to antigen-only controls (Figure $\mathbf{2 H}$ ). Alternatively, there was a two-fold increase in CD8 T cell proliferation with MPLA-stimulated GM-CSF BMDCs (Figure 2I). CD4 T cell proliferation significantly increased when mixed with CpG NP- and MPLA NPstimulated FLT3L BMDCs (Figure 2J). CD8 T cell proliferation significantly increased when mixed with FLT3L BMDCs stimulated with CpG, MPLA-CpG, and CpG+PUUC NPs (Figure 2K). Combination adjuvants synergistically or additively induced proinflammatory cytokine secretion by APCs in vitro while select adjuvants, including MPLA NP with GMCSF BMDCs and CpG-single and dual NP formulations with FLT3L BMDCs, increased CD8 $\mathrm{T}$ cell proliferation in vitro. We were motivated to evaluate whether these in vitro studies would predict how combination and single TLR- and RIG-I targeted adjuvants would improve SARS-CoV-2 subunit vaccination in vivo.

MPLA-PUUC NPs increase lung $T$ cell responses in mice following intranasal vaccination with SARS-CoV-2 stabilized spike protein

Given the protective role of mucosal immunity against respiratory virus SARS-CoV-2, ${ }^{36}$ we vaccinated mice intranasally with single and dual adjuvant-loaded NPs combined with either soluble (S-S) or NP-conjugated $S$ protein (NP-S). Mice were intranasally immunized with a primer on Day 0 and booster of the same dose on Day 28. Lungs were harvested and cell lysates restimulated with S-S and NP-S on Day 35. Lungs from mice immunized with MPLA+PUUC NP plus NP-S had significantly higher CD4 ${ }^{+} \mathrm{CD} 44^{+} \mathrm{T}$ cell populations with significant increases in IFNy and TNFa secretion when restimulated with $S$ peptide pools (Figures 3A-C). $\mathrm{CD} 69^{+} \mathrm{CD} 103^{+}$tissue-resident memory $\mathrm{T}$ cell populations were highest in mice vaccinated with MPLA NP and MPLA+PUUC NP plus S-S (Figure 3D). These cells exhibited a double negative CD4-CD8- phenotype, suggesting they could be $\gamma \delta$ cells, a subset of T cells enriched in epithelial and mucosal tissues that are activated in an MHC-independent manner. Intranasal vaccination with CpG, CpG-PUUC, R848-PUUC, or MPLA-CpG NP delivered with S-S failed to generate significant increases in percentages of T cells producing IFNy or TNFa (Figure S2A-K). In mice immunized with MPLA NP plus S-S, anti-S BAL fluid IgG and IgA and serum IgG levels were higher compared to mice in PUUC NP and MPLA+PUUC NP groups (Figures 2E-G). 
A

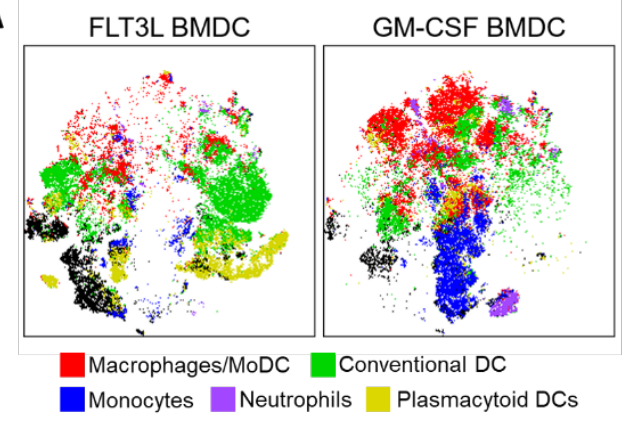

B

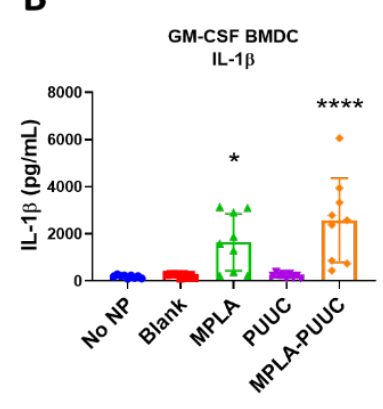

$\mathbf{F}$

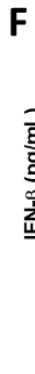

I

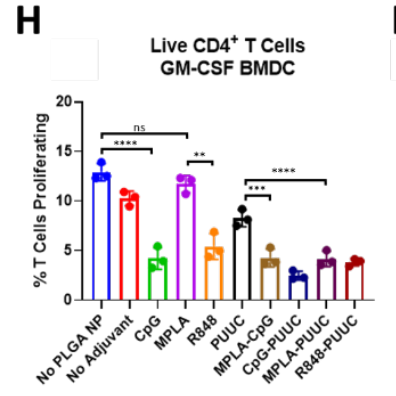

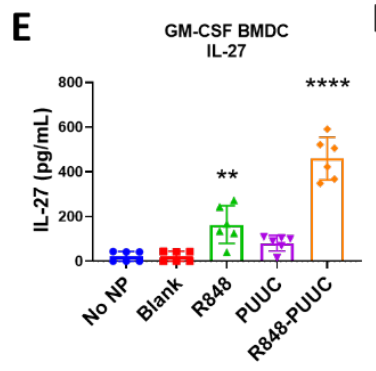
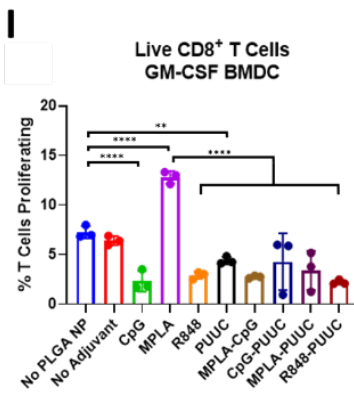

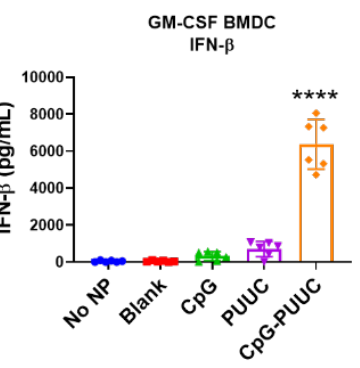

J

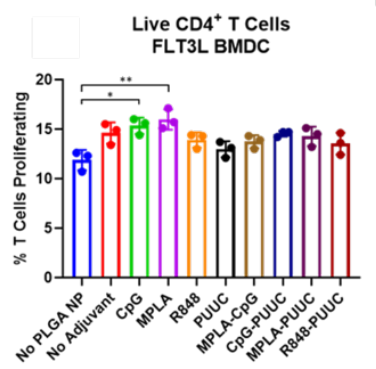

C

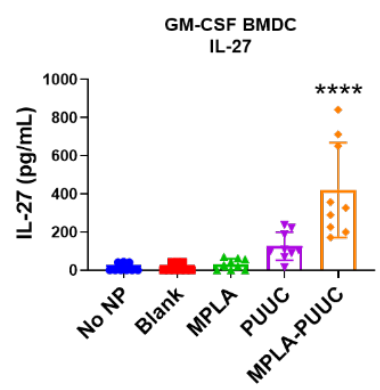

G

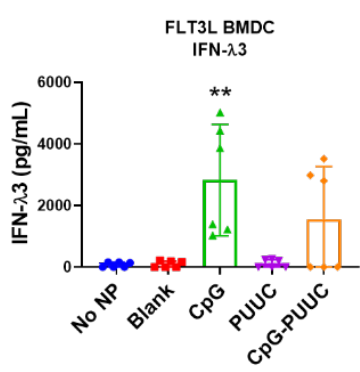

K

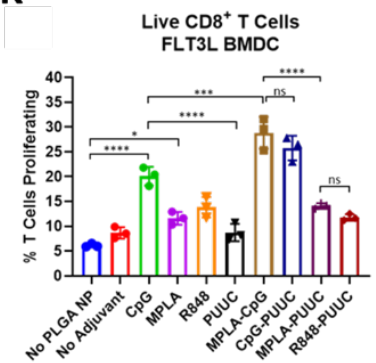

Figure 2: GM-CSF and FLT3L BMDCs secrete different cytokine profiles and activate CD8+ T cells in response to $\mathbf{S 1}$ protein with combination adjuvants. A) t-SNE plots of GM-CSF and FLT3L BMDCs with labeled clusters of APC subsets. Macrophages/Mo-DCs are CD64+ F4/80', Conventional DCs are $\mathrm{CD} 11 \mathrm{c}^{+} \mathrm{MHCI}{ }^{+} \mathrm{CD} 64^{10} \mathrm{~F} 4 / 80^{10}$, Monocytes are Ly6C ${ }^{\text {hi }} \mathrm{CD} 11 \mathrm{C}^{-}$, Neutrophils are Ly6G ${ }^{\text {hi }} \mathrm{Ly} \mathrm{C}^{+}$, plasmacytoid DCs are B220+ $\mathrm{Ly}_{6 C^{+}} \mathrm{CD} 11 \mathrm{C}^{+} \mathrm{MHCll}^{\mathrm{lo}}$. B-G) Cytokine concentrations $(\mathrm{pg} / \mathrm{mL})$ of IL-1 $\beta$, IL-27, IL-12p70, IFN- $\beta$, and IFN- $\lambda 3$ in supernatants of BMDC culture after incubation with adjuvanted NPs for $24 \mathrm{~h}$. $\mathrm{H}-\mathrm{I}$ ) Percentage of live CD3+CD4+ T cells or CD3+CD8+ T cells proliferating in presence of GM-CSF BMDCs, gated on diminished CFSE signal (Figure S1). "No Adjuvant" condition is non-adjuvant (blank) NPs. J-K) Percentage of live CD3+CD4+ T cells or CD3+CD8+ T cells proliferating in presence of FLT3L BMDCs. ${ }^{*} p<0.05$. ${ }^{* *} p<0.01,{ }^{* * *} p<0.001,{ }^{* * * *} p<0.0001$ based on a One-Way ANOVA and Tukey Test for multiple comparison. 

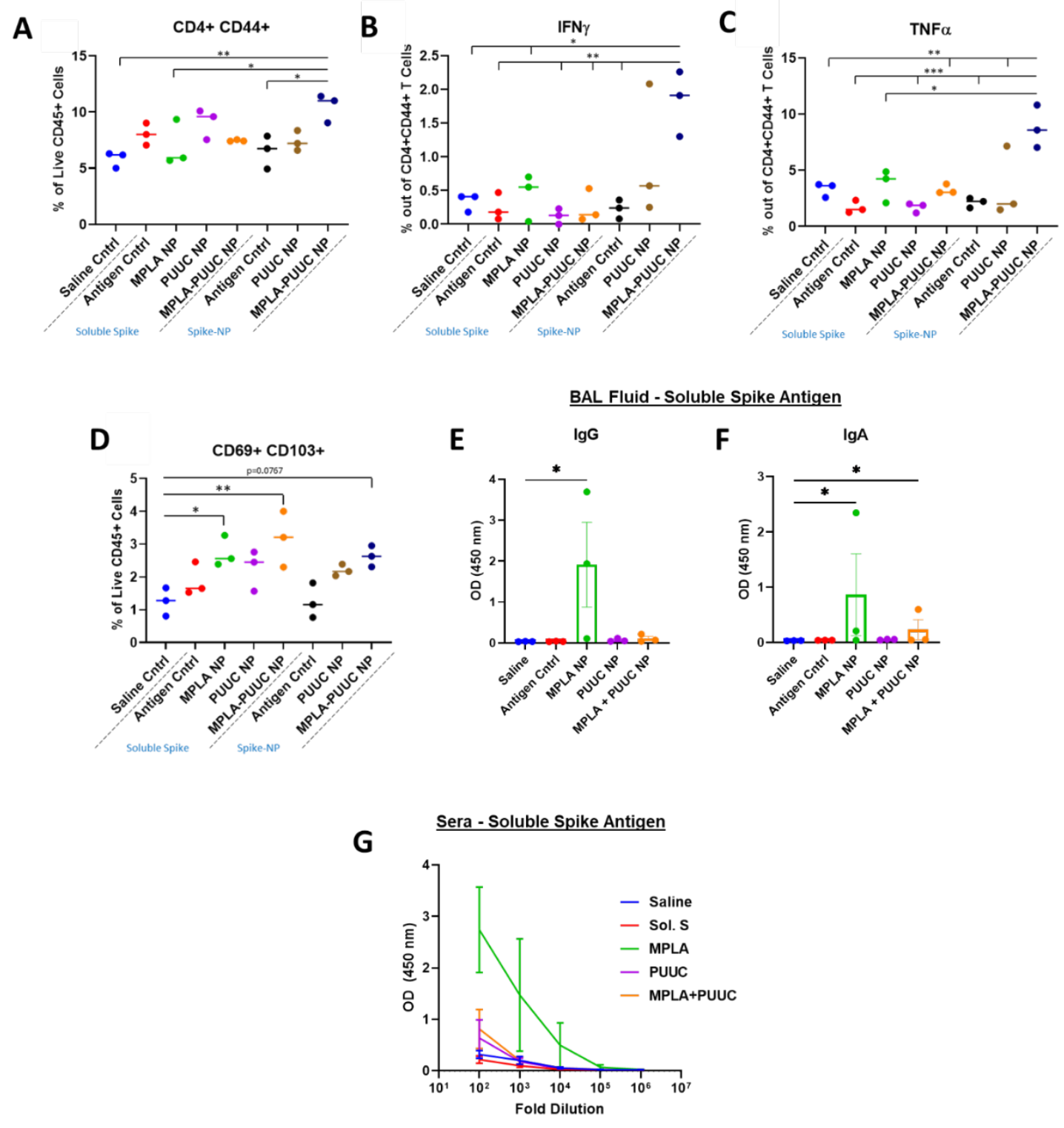

Figure 3: MPLA+PUUC NPs increase $T$ cell responses in the lung when delivered intranasally with spike protein. On days 0 (prime) and 28 (boost), female BALB/c mice were immunized by dropwise addition of saline and spike protein $(1 \mu \mathrm{g})$ with PLGA-PEI NPs $(4 \mathrm{mg})$ loaded with MPLA $(24 \mu \mathrm{g})$, PUUC $(17 \mu \mathrm{g})$, and MPLA+PUUC $(20 \mu \mathrm{g}, 17 \mu \mathrm{g})$. The same adjuvantNPs were combined with spike-conjugated NPs. Mice were euthanized and lungs were collected on day 35, one week after the booster. Lung cells were restimulated with spike peptide pools for $6 \mathrm{~h}$ and stained for analysis by flow cytometry. Percentages of cells expressing A) CD4+CD44+ out of CD45+ cells, B) IFNy+ out of CD4+CD44+ cells, C) TNFa+ out of CD4+CD44+ cells, D) CD69+CD103+ (tissue resident memory T cells) out of CD45+ cells. BAL fluid from vaccinated mice using soluble spike antigen was assayed for anti-spike E) $\lg$ G and F) $\lg$ A with ELISA. G) Sera were assayed for anti-spike IgG with ELISA (error bars represent the SEM). P values are * $p$ $\leq 0.05,{ }^{* *} p \leq 0.01,{ }^{* * *} p \leq 0.001$ calculated using A-D) One-way ANOVA with Tukey post-hoc test or E-F) Kruskal-Wallis with Dunn's post-hoc test for nonparametric data. 
PUUC NPs increase humoral responses in mice following intramuscular vaccination with various doses of SARS-CoV-2 stabilized spike protein

Because authorized COVID vaccines in the US are administered intramuscularly and antigen doses have been shown to impact T cell responses, ${ }^{37}$ we evaluated the ability of PUUC NPs to improve SARS-CoV-2 intramuscular protein subunit vaccines with different doses of $S$ protein. On Days 0 and 28, mice were immunized with $S$ protein at doses of 80,200 , or $1000 \mathrm{ng}$ with or without PUUC NPs. A cohort of mice received a mixed dose of $80 \mathrm{ng}$ on Day 0 and $1000 \mathrm{ng}$ on Day 28. Blood was drawn via the jugular vein of mice on Day 26 and cardiac puncture on Day 36 to quantify pre- and post-booster levels of antigen-specific IgG, which were higher in mice with PUUC NP-adjuvanted vaccines (Figure 4A-D). Post-booster anti-S IgG1 and IgG2a titers were simultaneously increased, especially for the $200 / 200,1000 / 1000$, and $80 / 1000$ (prime/boost) vaccination groups with PUUC NPs (Figure S3A-D). Neutralization of S protein with post-booster mouse sera was significantly detectible up in the $200 / 200,1000 / 1000$, and $80 / 1000 \mathrm{ng}$ prime/boost antigen concentrations adjuvanted with PUUC. Up to a 1000 -fold dilution of sera, neutralization of spike was significantly higher (ACE-2 A450 absorbance signal lower) in the 1000/1000 ng S mixed with PUUC NP group (Figure 4E).

By Day 36, percentages of GC BCL6 ${ }^{+} B 220^{+}$cells in popliteal lymph nodes significantly increased in the $200 / 200,1000 / 1000$, and $80 / 1000$ ng $S$ protein mice immunized with PUUC NP compared to non-adjuvanted and saline-injected control mice (Figure 4F). Similarly, GL7 ${ }^{+} \mathrm{B} 220^{+}$cell populations were significantly increased in mice immunized with 1000/1000 and 80/1000 ng S protein plus PUUC NP compared to controls (Figure 4G). PUUC NP with 80/80 ng S protein significantly decreased populations of B220- CXCR5 ${ }^{+}$ cells (T follicular helper, Tfh cells) in the dLNs (Figure 4H). In the spleen, PUUC NPs did not significantly change CD4 T cell population percentages (Figure S4A) nor IFN- $\gamma$, IL4, or TNF- $\alpha$ secretion by CD4 T cells (Figure S4B-D). CD8+ T cell populations and secretion of Granzyme B and TNF- $\alpha$ also did not change (Figure S4I-J, L, M-N, P). Interestingly, CD8+ T cell secretion of IFNy decreased in mice receiving PUUC NPs (Figure S4K, O). 
A

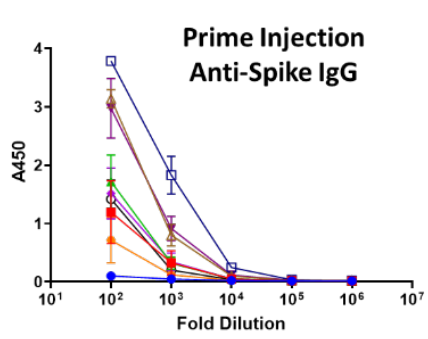

C

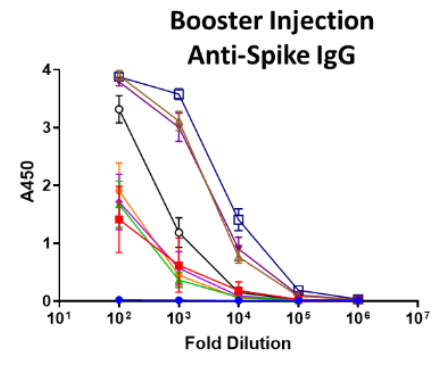

B

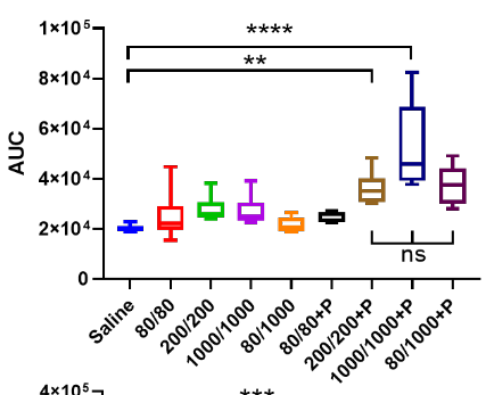

D

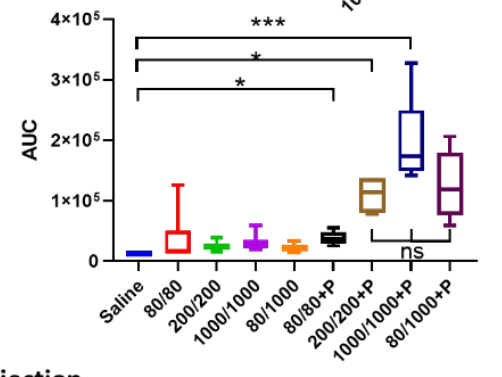

E

Booster Injection Neutralizing Anti-Spike Ig

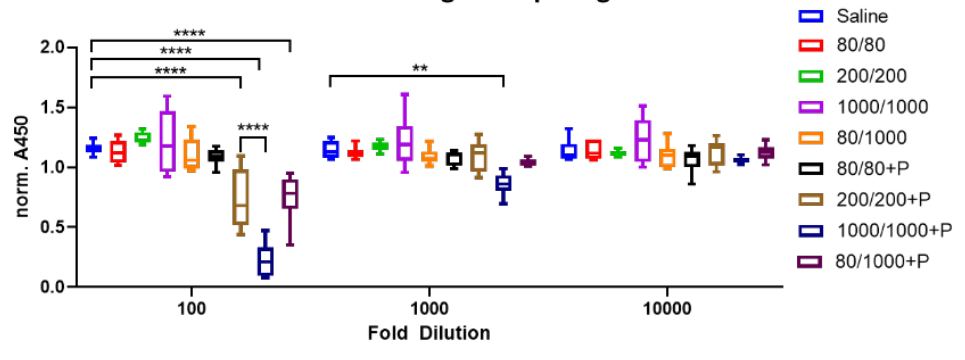

Popliteal Lymph Node Germinal Center B Cells

$\mathbf{F}$

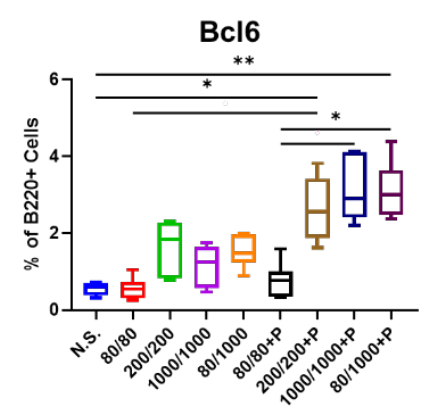

G

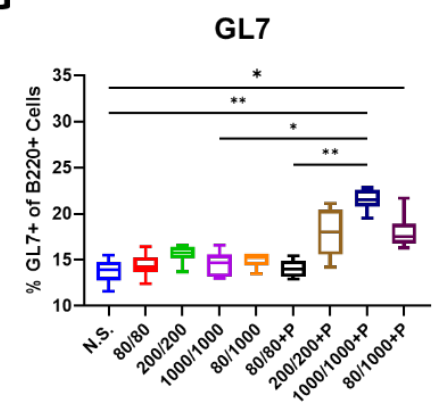

H

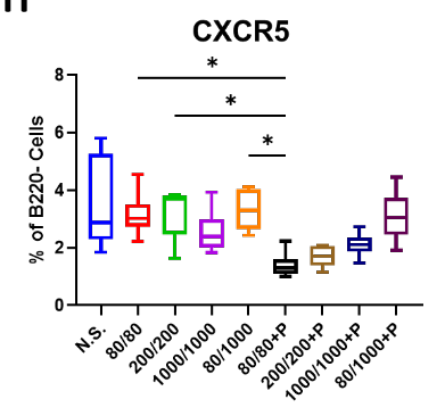

Figure 4: PUUC NPs delivered intramuscularly with spike protein enhance humoral responses. Female BALB/c mice were immunized by intramuscular injection into both tibialis anterior muscles at day 0 (prime) with saline or soluble spike protein at $80 \mathrm{ng}, 200 \mathrm{ng}, 1000 \mathrm{ng}$ with or without adjuvant-NPs (4 mg) loaded with PUUC (20 ng). Peripheral blood was drawn on day 26. On day 28, mice were injected with the same, except that half of the $80 \mathrm{ng}$ prime cohort were given a $1000 \mathrm{ng}$ booster. Mice were euthanized on day 36 for harvesting blood, splenocytes, and popliteal LNs. A) Anti-spike IgG in pre-booster sera at various dilutions measured by absorbance at 450 during ELISA assays and B) comparison of area under the curve (AUC). CD) Anti-spike lgG in post-booster sera measured by absorbance at 450 and comparison of AUC. 
E) Neutralizing anti-spike antibody levels, quantified with absorbance at $450 \mathrm{~nm}$ in a modified ELISA with biotinylated ACE-2 biotin. For neutralizing antibody quantification, absorbance was normalized to a blank well in each row of a 384 well plate to correct for plate-based effects. Lower absorbance values indicate higher neutralizing antibody levels. Percentages of cells expressing F) Bcl6+ out of B220+ cells, G) GL7+ out of B220+ cells and H) CXCR5+ out of B220- cells from the combined popliteal lymph nodes. B,D) Normality was assessed with the Kolmogorov-Smirnov test. Statistical significance was determined with the Kruskal-Wallis test and Dunn's post-hoc test for multiple comparisons. E-H) Statistical significance calculated with One-Way ANOVA and Tukey post-hoc test. ${ }^{*} p \leq 0.05,{ }^{* *} p \leq 0.01,{ }^{* * *} p \leq 0.001,{ }^{* * * *} p \leq 0.0001$ for all graphs.

Combination adjuvant MPLA+PUUC NPs do not increase humoral responses to intramuscular vaccination with SARS-CoV-2 spike protein compared to single adjuvant PUUC NPS

MPLA+PUUC NP increased lung cellular responses after intranasal $S$ protein subunit vaccination and antigen-specific $\lg G$ was not significantly different between the $1000 / 1000$ and $80 / 1000 \mathrm{ng} S$ protein intramuscular PUUC-adjuvanted vaccination groups. Therefore, we evaluated if MPLA+PUUC NP mixed with 80/1000 ng S-protein would enhance immune responses compared to single adjuvants. Mice were immunized with S protein mixed with MPLA NPs, PUUC NPs, and MPLA+PUUC NPs on Days 0 and 28. Pre- and post-booster antigen-specific IgG levels most significantly increased in mice vaccinated with $80 \mathrm{ng} S$ protein plus PUUC NP (Figure 5A-D). Neutralization of S protein was detectable with post-booster sera of PUUC NP-vaccinated mice up to a 100-fold dilution and MPLA+PUUC NP-vaccinated mice up to a 10,000-fold dilution (Figure 5E). In the draining popliteal LNs, B220+ cell percentage increased approximately $1.5 x$ in the MPLA NP group (Figure 5F). Out of $\mathrm{B}^{220^{+}}$cells, $\mathrm{BCL}^{+}$and $\mathrm{GL}^{+}$percentages significantly increased in the MPLA, PUUC, and MPLA+PUUC NP groups relative to the saline control group, and in the PUUC NP group relative to the antigen-only control group (Figure 5G-H). Out of B220- cells, the CXCR5 ${ }^{+}$population significantly increased in the PUUC and MPLA+PUUC NP groups compared to the saline control and in the MPLA+PUUC NP group relative to the antigen-only control group (Figure 5I).

In an analogous experiment using NP-S as antigen (1000/1000 ng S protein) instead of soluble protein, MPLA+PUUC NPs did not lead to a significant increase in anti-spike total IgG before or after a booster injection. Indeed, PUUC-NPs were associated with a decrease in IgG before a booster and both PUUC-NPs and MPLA+PUUC-NPs were associated with a decrease after a booster (Figure S5). 
A

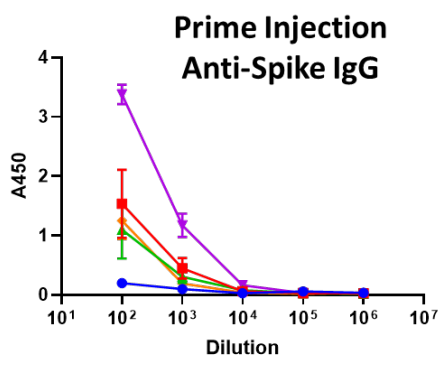

- Saline Cntrl

$\rightarrow$ Antigen Cntrl

$\rightarrow$ MPLANP

$\rightarrow$ PUUC NP

$\because$ MPLA-PUUC NP

B

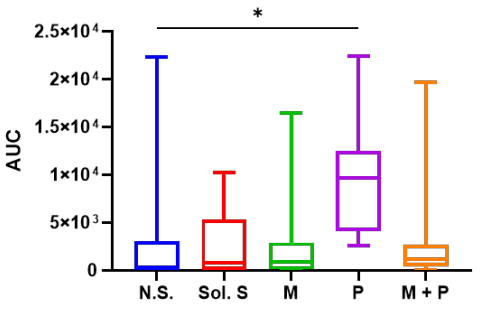

Booster Injection

C Anti-Spike IgG

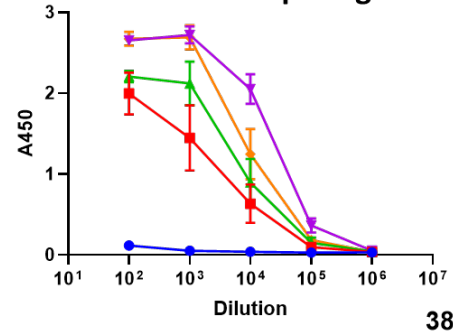

384-well Neutralization

$\mathbf{E}$

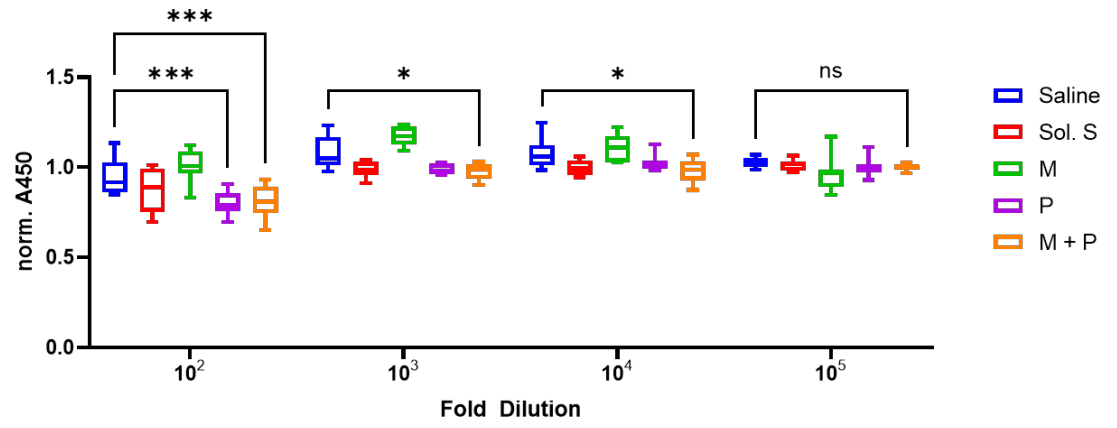

$\mathbf{F}$

B220

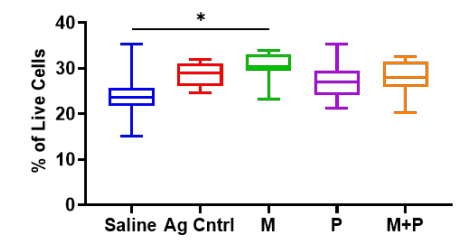

H

GL7

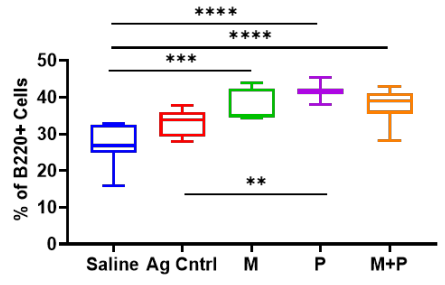

G

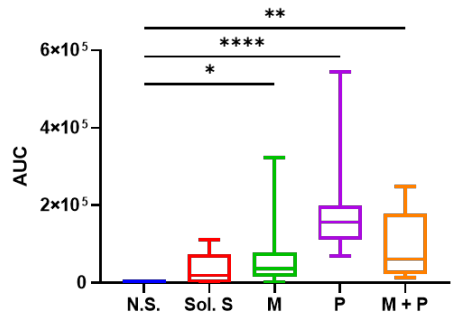

Figure 5. MPLA-PUUC NPs do not increase humoral responses to intramuscular spike protein subunit vaccine compared to PUUC NPs. Female BALB/c mice were immunized by intramuscular injection in the bilateral tibialis anterior muscles on day 0 with saline and spike 
protein (80 $\mathrm{ng}$ ) with or without adjuvant-NPs (4 mg) loaded with MPLA (24 $\mu \mathrm{g})$, PUUC (20 $\mu \mathrm{g})$, or MPLA+PUUC (24 $\mu \mathrm{g}, 20 \mu \mathrm{g})$. Pre-booster sera was collected on day 26 . On day 28, mice were boosted with either saline, spike protein (1000 ng), with adjuvant-NPs. Mice were euthanized on day 36 , and post-booster sera and popliteal lymph nodes were collected. A) Total anti-spike IgG quantified by absorbance at $450 \mathrm{~nm}$ during ELISA assay and B) AUC calculated for each dilution curve. C) Total anti-spike IgG in post-booster sera and D) AUC for each dilution curve. E) Neutralizing anti-spike antibodies quantified with absorbance at $450 \mathrm{~nm}$ using modified ELISA assay with biotinylated ACE-2 protein. Lower absorbance values indicate higher neutralizing antibody levels. Absorbance values normalized to blank wells in each row of 384 well plate to correct for plate-based effects. F) B220+ out of lymph node cells, G) Bcl6+ out of B220+ cells, H) GL7+ out of B220+ cells and I) CXCR5+ out of B220- cells from the combined popliteal lymph nodes. B,D) Normality was assessed with the Kolmogorov-Smirnov test. Statistical significance was determined with the Kruskal-Wallis test and Dunn's post-hoc test for multiple comparisons. E-I) Statistical significance calculated with One-Way ANOVA and Tukey post-hoc test. * $p \leq 0.05$, ${ }^{* *} p \leq 0.01,{ }^{* * *} p \leq 0.001,{ }^{* * * *} p \leq 0.0001$ for all graphs.

\section{DISCUSSION}

In response to studies revealing PAMP combinations synergistically and complementarily enhance immunity, we investigated the singular and combined effects of nanoparticledelivered TLR and RIG-I agonists on cellular and humoral immune responses against SARS-CoV-2 spike protein. Our data suggest MPLA plus PUUC has the potential to be an effective adjuvant formulation for SARS-CoV-2 protein subunit vaccines and is supported by evidence that the SARS-CoV-2 virus interacts with both TLR4 and RIG-I during natural infection. ${ }^{38,39}$ In vitro data showed MPLA+PUUC, R848+PUUC, and CpG+PUUC PLGA-PEI NPs stimulate more GM-CSF BMDC proinflammatory cytokine secretion (IL-1 $\beta$, IL-12p70, IFN- $\beta$ ) than single adjuvant controls, and MPLA NPs and CpG-containing NPs (i.e., CpG, CpG+PUUC) maintain or induce CD8+ T cell proliferation with GM-CSF or FLT3L BMDCs, respectively. GM-CSF BMDCs and FLT3L BMDCs, both widely used for evaluating APC maturation in vitro, had contradicting responses to TLR and RIG-I agonists most likely because the cultures are comprised of different APC populations (monocytes, monocyte-derived macrophages, and DCs). Consistent with previous reports, we found that GM-CSF BMDCs were primarily composed of a heterogeneous population of monocytes, monocyte-derived macrophages, monocytederived DCs, and neutrophils. ${ }^{34}$ FLT3L BMDCs contained higher percentages of conventional DCs and plasmacytoid DCs. ${ }^{40}$ Interestingly, cells derived from the Ly6C ${ }^{\text {hi }}$ monocyte lineage are known to secrete IL-27 in vivo in response to subunit vaccination with multiple TLR adjuvants, which may explain higher IL-27 production by GM-CSF BMDCs compared to FLT3L BMDCs. ${ }^{41}$

In GM-CSF BMDC culture, MPLA+PUUC NPs induced significant IL-27 production while single-adjuvant MPLA NPs and PUUC NPs were not as potent. IL-27 is a heterodimer of 
the EBI3 and p28 subunits, ${ }^{42}$ and TLR4 ligation by MPLA generates EBI3 and some p28. ${ }^{43}$ PUUC signaling through RIG-I induces the production of type I interferons, which strongly promote the transcription of p28 through the binding of IRF-1 to IRSEs. ${ }^{29,43}$ It is plausible that IL-27 synthesis in response to MPLA+PUUC is due to the increase in p28 to PUUC-induced type I interferons combined with the increase in p28 and EBI3 to MPLA signaling. Furthermore, possibly due to IL-27-induced expression of $\mathrm{T}$ cell inhibitory receptors on APCs such as PD-L1, LAG-3, CTLA-4, and TIGIT, 44 MPLA+PUUC NPs and R848+PUUC NPs decreased T cell proliferation with GM-CSF BMDCs compared to controls without adjuvant. Alternatively, MPLA NPs increased CD8+ T cell proliferation when cocultured with both GM-CSF and FLT3L BMDCs. This could be explained by the expression of TLR4-induced costimulatory molecules on APCs in the absence of IL-27induced $\mathrm{T}$ cell inhibitory receptors. ${ }^{45}$

Due to observed increases in proinflammatory cytokine production in vitro, we predicted that MPLA+PUUC NPs, R848+PUUC NPs, and CpG+PUUC NPs would elicit stronger cellular and humoral immune responses in vivo to SARS-CoV-2 S protein subunit vaccines compared to single-adjuvanted or non-adjuvanted vaccines. Previous studies have reported delivering antigens and adjuvants in separate PLGA particles can improve or attain the same immune responses compared to co-delivered antigens and adjuvants. Therefore, we opted to deliver antigen and adjuvant separately for these studies. ${ }^{46,47}$ Also, because APCs innately recognize the particulate state of microbes, we tested adjuvants with both NP-conjugated (NP-S) and soluble (S-S) SARS-CoV-2 S protein antigens. ${ }^{48}$ CpG-PUUC and R848-PUUC NP delivered with S-S failed to generate significant increases in percentages of $\mathrm{T}$ cells producing IFNy or TNFa. A previous study showed that intramuscular vaccination with R848-PUUC NPs induced lymphopenia, possibly due to the overproduction of type I IFN. ${ }^{29}$ Studies utilizing CpG 1018 or TLR7/8 agonists in SARS-CoV-2 S protein subunit vaccines combine the TLR agonists with alum, which alone traditionally induces a Th2-type immune response. ${ }^{49-51}$

Intranasal vaccination of mice with MPLA+PUUC NPs plus NP-S protein increased $\mathrm{CD}_{4}{ }^{+} \mathrm{CD} 4 \mathrm{~T}$ cell populations with IFN- $\gamma$ and TNF- $\alpha$ responses in lung cells during restimulation with SARS-CoV-2 $S$ protein peptide pools. CD44 is a marker that distinguishes effector and memory $T$ cells from naïve subsets. ${ }^{52}$ Because these CD44 ${ }^{+}$ CD4 $\mathrm{T}$ cells were enriched for intracellular IFN- $\gamma$ and TNF- $\alpha$, our results suggest that these cells were polarized towards a Th1 effector phenotype, which may be essential for controlling SARS-CoV-2 infection in humans. ${ }^{53-55}$ Routhu et al. intranasally administered a SARS-CoV-2 RBD trimer subunit vaccine with alum plus 3M-052 (TLR7/8 agonist) in rhesus macaques and also observed a Th1-biased response, but no detectable antigenspecific CD8+ $\mathrm{T}$ cell response. ${ }^{50}$ A preclinical study by Jangra et al. investigating a nanoemulsion +/- RIG-I agonist (IVT DI) adjuvanted SARS-CoV-2 S1 subunit intranasal vaccine also observed increased IFNy secreted by splenocytes and dLN cells, indicating 
a Th1 response. ${ }^{56}$ Cell populations bearing tissue-resident memory $\mathrm{T}$ cell markers $\left(\mathrm{CD}{ }^{+} \mathrm{CD} 103^{+}\right)$also increased. Because these $\mathrm{T}$ cells were $\mathrm{CD}^{-}{ }^{-} \mathrm{CD}^{-}$we speculate they could be $ү \delta \mathrm{T}$ cells, a subset of T cells enriched in epithelial and mucosal tissues that are activated in an $\mathrm{MHC}$-independent manner ${ }^{57,58}$. Unexpectedly, in post-booster BAL fluid and sera, antigen-specific $\lg G$ and $\lg A$ were present following intranasal vaccination with MPLA NPs plus S-S, but not with PUUC NPs or MPLA+PUUC NPs. Jangra et al. did observe IgG in the BAL fluid and sera but also administered a 15- $\mu \mathrm{g}$ S1

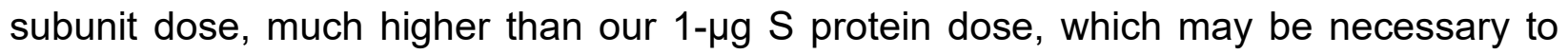
induce humoral responses. The addition of RIG-I agonist to their nanoemulsion adjuvant also did not enhance humoral responses against S1 subunit protein. ${ }^{56}$

Intramuscular vaccination with NP-S or S-S plus adjuvant induced very different antigenspecific immune responses compared to intranasal vaccination. Antigen-specific lgG response in mice intramuscularly immunized with NP-S was minimal; IgG was only detectable at 1:100 dilution post-booster in formulations without adjuvant (Figure S5). Conversely, in intramuscular vaccinations with S-S, PUUC NPs generated significant antigen-specific IgG in sera, pre- and post-booster. Post-booster sera from mice immunized with S-S also contained antibodies neutralizing $S$ protein, meaning ACE-2 binding was reduced; these results are noteworthy because SARS-CoV-2 enters respiratory endothelial cells via endocytosis following ACE-2 binding. ${ }^{59,60}$ We also found that S-S plus PUUC NP was associated with increased GC (GC) BCL6 ${ }^{+}$and $\mathrm{GL7} 7^{+} \mathrm{B}$ cells in the dLNs. Because the GC reaction produces high-affinity isotype switched antibodies by both plasma cells and memory B cells, ${ }^{29,61}$ we suspect that the increased $\mathrm{GC}$ response associated with PUUC NPs explains the increase in antigen-specific-spike IgG and neutralizing antibodies. This $\mathrm{GC}$ reaction may be a response to early innate inflammation associated with PUUC, such as endothelial cell activation which has been reported with RIG-I ligation in viral models, ${ }^{62-64}$ and the APC activation which we have observed in our in vitro studies. Because both IgG1 and IgG2a were increased following intramuscular vaccination with PUUC NPs and S-S, we do not attribute the overall increase in antibodies to either a Th1- or Th2-mediated bias. ${ }^{65}$ This finding contrasts with previous studies that have shown a Th2 bias with RIG-I-based adjuvants following intraperitoneal vaccination with influenza VLPs. ${ }^{66}$

Zhou et al. showed that the inclusion of alum in SARS-CoV S protein subunit vaccines reduced the effective antigen dose tenfold. In mice, the alum-adjuvanted $5-\mu \mathrm{g} S$ protein subunit vaccine produced twice as many neutralizing antibodies as the nonadjuvanted $50-\mu g$ S protein subunit vaccine.$^{67}$ Interestingly in our studies, the 80 -ng primer and 1000 ng S protein boost produced similar increases in GC B cell populations compared to the 1000-ng primer and 1000-ng boost, indicating an opportunity for antigen dose sparing with the inclusion of PUUC as an adjuvant. MPLA+PUUC NPs plus S-S reduced antigenspecific IgG following the first injection and performed equally to PUUC NPs following the 
booster injection. MPLA+PUUC NPs and PUUC NPs increased BCL6 ${ }^{+}$and $\mathrm{GL7}^{+} \mathrm{B}$ cell populations. MPLA+PUUC NPs did significantly induce more neutralizing antibody activity compared to PUUC NPs or MPLA NPs alone, and increased B220-CXCR5 ${ }^{+}$cell populations (Tfh markers).

Compared to trials with soluble antigen, it is interesting that MPLA+PUUC NPs failed to elicit a strong anti-spike IgG response when NP-conjugated spike was used instead. This effect could be explained by a hindered ability of NP-conjugated molecules encounter B cells independently, as particles $>200 \mathrm{~nm}$ in diameter drain less efficiently to lymphatics. ${ }^{68}$ Additionally, given that particle surface topology alone can trigger innate immune responses, particulate antigen might be sufficiently immunogenic enough that when combined with PUUC or MPLA+PUUC, lymphocytes become anergic, giving a reduced adaptive response. 69

\section{CONCLUSION}

Our results demonstrate that polymer-NP delivery of the RIG-I agonist PUUC), and the TLR4 agonist MPLA, increases immune responses to SARS-CoV-2 spike protein subunit vaccines compared to non-adjuvanted vaccines. MPLA+PUUC NP also elicited differential cellular and humoral responses against SARS-CoV-2 spike protein depending on the APCs encountered (GM-CSF versus FLT3L-derived), route of administration (intramuscular versus intranasal), and delivery-platform of the $\mathrm{S}$ protein (soluble versus NP-conjugated). We show that I.N, administration with PUUC+MPLA NPs induces memory $T$ cell responses in the lung while MPLA NPs induces local $\lg A$ and $\lg G$ responses in the lung. In contrast, I.M. administration induces robust systemic total and neutralizing antibody responses against the SARS-CoV-2 spike protein, Future investigations should examine whether a combination of I.N. and I.M vaccination can produce balanced systemic and lung-specific protective immunity against SARS-CoV-2 challenge and improve vaccine durability and protection against infection and transmission.

\section{ACKNOWLEDGMENTS}

We wish to acknowledge the core facilities at the Parker $H$. Petit Institute for Bioengineering and Bioscience at the Georgia Institute of Technology for the use of their shared equipment, services, and expertise. These facilities include: the Biopolymer Characterization Core for the preparation of PLGA particles, the Engineered Biosystems Building Physiological Research Laboratory for animal experiments, and the Cellular Analysis and Cytometry Core for flow cytometry experiments. Research reported in this publication was supported in part by the Pediatrics/Winship Flow Cytometry Core of 
Winship Cancer Institute of Emory University, Children's Healthcare of Atlanta and $\mathrm{NIH} / \mathrm{NCl}$ under award number P30CA138292. The content is solely the responsibility of the authors and does not necessarily represent the official views of the National Institutes of Health. This work was partially funded by NIH/NIAID grant U01-AI124270-02 to KR, funds from the Georgia Tech Foundation to $\mathrm{KR}$, the National Science Foundation Graduate Research Fellowship to AA, the NIH T32 Cellular and Tissue Engineering training fellowship (NIH grant T32-GM0843) to $A A$ and $A B$, and the Robert $A$. Milton Chaired Professorship to KR.

\section{CONTRIBUTIONS}

A.A., B.P., P.P., and K.R. conceptualized the idea and methodology. K.R. acquired the funding. A.A. was the project administrator and supervisor for iso-MLR assays and A.A. and M.K. were joint supervisors for all in vivo studies. P.P. and B.P. helped A.A. and M.K. conduct in vivo vaccinations. B.P. supervised adjuvant formulation and NP synthesis. A.A., M.K., A.B., and B.P. curated the data. A.A. and M.K. conducted formal analyses. A.A., M.K., B.P., A.B., P.P., C.V., R.J., J.H., C.S., L.K., M.A.O., and D.F. harvested and processed blood and tissue samples from in vivo studies for further investigation. A.A., M.K. and B.P. wrote the manuscript. K.R. revised the manuscript. All authors reviewed the final manuscript and submitted comments.

\section{CONFLICTS OF INTEREST}

There are no conflicts of interest to disclose.

\section{REFERENCES}

1 WHO COVID-19 Dashboard, <https://covid19. who.int/> (2021).

2 R\&D Blue Print. COVID-19 vaccine tracker and landscape. (2021).

3 Food and Drug Administration. (2021).

4 Awate, S., Babiuk, L. A. B. \& Mutwiri, G. Mechanisms of action of adjuvants. Frontiers in immunology 4, 114 (2013).

5 Reed, S. G., Orr, M. T. \& Fox, C. B. Key roles of adjuvants in modern vaccines. Nature medicine 19, 1597-1608 (2013).

6 Saravia, J., Chapman, N. M. \& Chi, H. Helper T cell differentiation. Cellular \& molecular immunology 16, 634-643 (2019).

7 Maddur, M. S. \& Bayry, J. B cells drive Th2 responses by instructing human dendritic cell maturation. Oncoimmunology 4, e1005508 (2015). 
8 Ionescu, L. \& Urschel, S. Memory B cells and long-lived plasma cells. Transplantation 103, 890-898 (2019).

9 Rehwinkel, J. \& Gack, M. U. RIG-I-like receptors: their regulation and roles in RNA sensing. Nature Reviews Immunology 20, 537-551 (2020).

10 Schnell, G., Loo, Y.-M., Marcotrigiano, J. \& Gale Jr, M. Uridine composition of the poly-U/UC tract of HCV RNA defines non-self recognition by RIG-I. PLoS pathogens 8, e1002839 (2012).

11 Lu, Y.-C., Yeh, W.-C. \& Ohashi, P. S. LPS/TLR4 signal transduction pathway. Cytokine 42, 145-151 (2008).

12 Pålsson-McDermott, E. M. \& O'Neill, L. A. Signal transduction by the lipopolysaccharide receptor, Toll-like receptor-4. Immunology 113, 153-162 (2004).

13 Casella, C. R. \& Mitchell, T. C. Putting endotoxin to work for us: monophosphoryl lipid $A$ as a safe and effective vaccine adjuvant. Cellular and molecular life sciences 65, 3231-3240 (2008).

14 Hemmi, H. et al. Small anti-viral compounds activate immune cells via the TLR7 MyD88-dependent signaling pathway. Nature immunology 3, 196-200 (2002).

15 Ashkar, A. A. \& Rosenthal, K. L. Toll-like receptor 9, CpG DNA and innate immunity. Current molecular medicine 2, 545-556 (2002).

16 Warner, N. \& Núñez, G. MyD88: a critical adaptor protein in innate immunity signal transduction. The Journal of Immunology 190, 3-4 (2013).

17 Kawasaki, T. \& Kawai, T. Toll-like receptor signaling pathways. Frontiers in immunology 5, 461 (2014).

18 Takeda, K. \& Akira, S. in Seminars in immunology. 3-9 (Elsevier).

19 Saito, T., Owen, D. M., Jiang, F., Marcotrigiano, J. \& Gale Jr, M. Innate immunity induced by composition-dependent RIG-I recognition of hepatitis C virus RNA. Nature 454, 523-527 (2008).

20 Moliva, J. I., Turner, J. \& Torrelles, J. B. Immune responses to bacillus CalmetteGuérin vaccination: why do they fail to protect against Mycobacterium tuberculosis? Frontiers in immunology 8, 407 (2017).

21 Lund, J. M. et al. Recognition of single-stranded RNA viruses by Toll-like receptor 7. Proceedings of the National Academy of Sciences 101, 5598-5603 (2004).

22 Weber, F., Wagner, V., Rasmussen, S. B., Hartmann, R. \& Paludan, S. R. Doublestranded RNA is produced by positive-strand RNA viruses and DNA viruses but not in detectable amounts by negative-strand RNA viruses. Journal of virology 80 , 5059-5064 (2006).

23 Kell, A. M. \& Gale Jr, M. RIG-I in RNA virus recognition. Virology 479, 110-121 (2015). 
24 Jensen, S. \& Thomsen, A. R. Sensing of RNA viruses: a review of innate immune receptors involved in recognizing RNA virus invasion. Journal of virology 86, 29002910 (2012).

25 Shirato, K. \& Kizaki, T. SARS-CoV-2 spike protein S1 subunit induces proinflammatory responses via toll-like receptor 4 signaling in murine and human macrophages. Heliyon 7, e06187 (2021).

26 Choudhury, A. \& Mukherjee, S. In silico studies on the comparative characterization of the interactions of SARS-CoV-2 spike glycoprotein with ACE-2 receptor homologs and human TLRs. Journal of medical virology 92, 2105-2113 (2020).

27 Zhao, Y. et al. SARS-CoV-2 spike protein interacts with and activates TLR41. Cell research, 1-3 (2021).

28 Pradhan, P. et al. TRAF6-IRF5 kinetics, TRIF, and biophysical factors drive synergistic innate responses to particle-mediated MPLA-CpG co-presentation. Science advances 7, eabd4235 (2021).

29 Toy, R. et al. TLR7 and RIG-I dual-adjuvant loaded nanoparticles drive broadened and synergistic responses in dendritic cells in vitro and generate unique cellular immune responses in influenza vaccination. Journal of Controlled Release 330, 866-877 (2021).

30 Stadnytskyi, V., Bax, C. E., Bax, A. \& Anfinrud, P. The airborne lifetime of small speech droplets and their potential importance in SARS-CoV-2 transmission. Proceedings of the National Academy of Sciences 117, 11875-11877 (2020).

$31 \mathrm{Li}, \mathrm{S}$. et al. SARS-CoV-2: Mechanism of infection and emerging technologies for future prospects. Reviews in Medical Virology 31, e2168 (2021).

32 Trougakos, I. P. et al. Insights to SARS-CoV-2 life cycle, pathophysiology, and rationalized treatments that target COVID-19 clinical complications. Journal of Biomedical Science 28, 1-18 (2021).

33 Leleux, J. A., Pradhan, P. \& Roy, K. Biophysical attributes of CpG presentation control TLR9 signaling to differentially polarize systemic immune responses. Cell reports 18, 700-710 (2017).

34 Helft, J. et al. GM-CSF mouse bone marrow cultures comprise a heterogeneous population of CD11c+ MHCII+ macrophages and dendritic cells. Immunity 42, 1197-1211 (2015).

35 Corbett, K. S. et al. Evaluation of the mRNA-1273 vaccine against SARS-CoV-2 in nonhuman primates. New England Journal of Medicine 383, 1544-1555 (2020).

36 Russell, M. W., Moldoveanu, Z., Ogra, P. L. \& Mestecky, J. Mucosal immunity in COVID-19: a neglected but critical aspect of SARS-CoV-2 infection. Frontiers in Immunology 11, 3221 (2020). 
37 Billeskov, R., Beikzadeh, B. \& Berzofsky, J. A. The effect of antigen dose on T celltargeting vaccine outcome. Human vaccines \& immunotherapeutics $15,407-411$ (2019).

38 Bhattacharya, M. et al. Immunoinformatics approach to understand molecular interaction between multi-epitopic regions of SARS-CoV-2 spike-protein with TLR4/MD-2 complex. Infection, Genetics and Evolution 85, 104587 (2020).

39 Yamada, T. et al. RIG-I triggers a signaling-abortive anti-SARS-CoV-2 defense in human lung cells. Nature Immunology, 1-9 (2021).

40 Brasel, K., De Smedt, T., Smith, J. L. \& Maliszewski, C. R. Generation of murine dendritic cells from flt3-ligand-supplemented bone marrow cultures. Blood, The Journal of the American Society of Hematology 96, 3029-3039 (2000).

41 Kilgore, A. M. et al. IL-27p28 production by XCR1+ dendritic cells and monocytes effectively predicts adjuvant-elicited CD8+ T cell responses. ImmunoHorizons 2 , 1-11 (2018).

42 Carl Jr, J. W. \& Bai, X.-F. IL27: its roles in the induction and inhibition of inflammation. International journal of clinical and experimental pathology 1, 117 (2008).

43 Pirhonen, J., Siren, J., Julkunen, I. \& Matikainen, S. IFN- $\alpha$ regulates Toll-like receptor-mediated IL-27 gene expression in human macrophages. Journal of leukocyte biology 82, 1185-1192 (2007).

44 DeLong, J. H. et al. IL-27 and TCR stimulation promote T cell expression of multiple inhibitory receptors. ImmunoHorizons 3, 13-25 (2019).

45 Shen, H., Tesar, B. M., Walker, W. E. \& Goldstein, D. R. Dual signaling of MyD88 and TRIF is critical for maximal TLR4-induced dendritic cell maturation. The Journal of Immunology 181, 1849-1858 (2008).

46 Kasturi, S. P. et al. Programming the magnitude and persistence of antibody responses with innate immunity. Nature 470, 543-547 (2011).

47 Kazzaz, J. et al. Encapsulation of the immune potentiators MPL and RC529 in PLG microparticles enhances their potency. Journal of controlled release 110, 566-573 (2006).

48 Moyer, T. J., Zmolek, A. C. \& Irvine, D. J. Beyond antigens and adjuvants: formulating future vaccines. The Journal of clinical investigation 126, 799-808 (2016).

49 Kuo, T.-Y. et al. Development of CpG-adjuvanted stable prefusion SARS-CoV-2 spike antigen as a subunit vaccine against COVID-19. Scientific reports 10, 1-10 (2020).

50 Routhu, N. K. et al. SARS-CoV-2 RBD trimer protein adjuvanted with Alum-3M052 protects from SARS-CoV-2 infection and immune pathology in the lung. Nature communications 12, 1-15 (2021). 
51 Brewer, J. M. et al. Aluminium hydroxide adjuvant initiates strong antigen-specific Th2 responses in the absence of IL-4-or IL-13-mediated signaling. The Journal of Immunology 163, 6448-6454 (1999).

52 Schumann, J., Stanko, K., Schliesser, U., Appelt, C. \& Sawitzki, B. Differences in CD44 surface expression levels and function discriminates IL-17 and IFN-Y producing helper T cells. PLoS One 10, e0132479 (2015).

53 Raphael, I., Nalawade, S., Eagar, T. N. \& Forsthuber, T. G. T cell subsets and their signature cytokines in autoimmune and inflammatory diseases. Cytokine 74, 5-17 (2015).

54 Sette, A. \& Crotty, S. Adaptive immunity to SARS-CoV-2 and COVID-19. Cell (2021).

55 Moderbacher, C. R. et al. Antigen-specific adaptive immunity to SARS-CoV-2 in acute COVID-19 and associations with age and disease severity. Cell 183, 9961012. e1019 (2020).

56 Jangra, S. et al. A Combination Adjuvant for the Induction of Potent Antiviral Immune Responses for a Recombinant SARS-CoV-2 Protein Vaccine. bioRxiv (2021).

57 Kang, S. et al. Tissue Resident Memory yठT Cells in Murine Uterus Expressed High Levels of IL-17 Promoting the Invasion of Trophocytes. Frontiers in Immunology 11 (2020).

58 Do, J.-s. et al. $ү \delta$ T cells coexpressing gut homing $\alpha 4 \beta 7$ and $\alpha E$ integrins define a novel subset promoting intestinal inflammation. The Journal of Immunology 198 , 908-915 (2017).

59 Zhou, P. et al. A pneumonia outbreak associated with a new coronavirus of probable bat origin. nature 579, 270-273 (2020).

60 Lan, J. et al. Structure of the SARS-CoV-2 spike receptor-binding domain bound to the ACE2 receptor. Nature 581, 215-220 (2020).

61 Gatto, D. \& Brink, R. The germinal center reaction. Journal of Allergy and Clinical Immunology 126, 898-907 (2010).

62 da Conceicao, T. M. et al. Essential role of RIG-I in the activation of endothelial cells by dengue virus. Virology 435, 281-292 (2013).

63 Kell, A. M., Hemann, E. A., Turnbull, J. B. \& Gale Jr, M. RIG-I-like receptor activation drives type I IFN and antiviral signaling to limit Hantaan orthohantavirus replication. PLoS pathogens 16, e1008483 (2020).

64 Asdonk, T. et al. Endothelial RIG-I activation impairs endothelial function. Biochemical and biophysical research communications 420, 66-71 (2012).

65 Holdsworth, S. R., Kitching, A. R. \& Tipping, P. G. Th1 and Th2 T helper cell subsets affect patterns of injury and outcomes in glomerulonephritis. Kidney international 55, 1198-1216 (1999). 
66 Beljanski, V. et al. Enhanced influenza virus-like particle vaccination with a structurally optimized RIG-I agonist as adjuvant. Journal of virology 89, 1061210624 (2015).

67 Zhou, Z. et al. A recombinant baculovirus-expressed S glycoprotein vaccine elicits high titers of SARS-associated coronavirus (SARS-CoV) neutralizing antibodies in mice. Vaccine 24, 3624-3631 (2006).

68 Schudel, A., Francis, D. M. \& Thomas, S. N. Material design for lymph node drug delivery. Nature Reviews Materials 4, 415-428 (2019).

69 Baranov, M. V., Kumar, M., Sacanna, S., Thutupalli, S. \& Van Den Bogaart, G. Modulation of immune responses by particle size and shape. Frontiers in immunology 11, 3854 (2020). 